Research Paper

\title{
Application of phototherapeutic-based nanoparticles in colorectal cancer
}

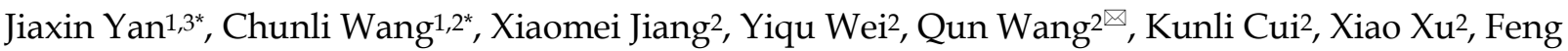 \\ Wang ${ }^{\bowtie}$, Lei Zhang ${ }^{2 \bowtie}$ \\ 1. Bioinformatics Center, School of Basic Medical Sciences, Henan University, Kaifeng 475004, China. \\ 2. School of Basic Medical Sciences, Henan University, Kaifeng 475004, China. \\ 3. School of Pharmacy, Henan University, Kaifeng Kaifeng 475004, China. \\ 4. Guangming Substation of Shenzhen Ecological Environment Monitoring Station, Shenzhen 518107, P. R. China. \\ * These authors contributed equally to this work. \\ $\bowtie$ Corresponding authors: Lei Zhang, zhlei@henu.edu.cn; Qun Wang, wangqun011@163.com; Feng Wang, 317646245@qq.com. \\ (C) The author(s). This is an open access article distributed under the terms of the Creative Commons Attribution License (https://creativecommons.org/licenses/by/4.0/). \\ See http://ivyspring.com/terms for full terms and conditions.
}

Received: 2021.01.28; Accepted: 2021.03.12; Published: 2021.04.02

\begin{abstract}
Colorectal cancer (CRC) is the third most commonly diagnosed malignancy and the second leading cause of cancer death, which accounts for approximately $10 \%$ of all new cancer cases worldwide. Surgery is the main method for treatment of early-stage CRC. However, it is not effective for most metastatic tumors, and new treatment and diagnosis strategies need to be developed. Photosensitizers (PSs) play an important role in the treatment of CRC. Phototherapy also has a broad prospect in the treatment of CRC because of its low invasiveness and low toxicity. However, most PSs are associated with limitations including poor solubility, poor selectivity and high toxicity. The application of nanomaterials in PSs has added many advantages, including increased solubility, bioavailability, targeting, stability and low toxicity. In this review, based on phototherapy, we discuss the characteristics and development progress of PSs, the targeting of PSs at organ, cell and molecular levels, and the current methods of optimizing PSs, especially the application of nanoparticles as carriers in CRC. We introduce the photosensitizer (PS) targeting process in photodynamic therapy (PDT), the damage mechanism of PDT, and the application of classic PS in CRC. The action process and damage mechanism of photothermal therapy (PTT) and the types of ablation agents. In addition, we present the imaging examination and the application of PDT / PTT in tumor, including (fluorescence imaging, photoacoustic imaging, nuclear magnetic resonance imaging, nuclear imaging) to provide the basis for the early diagnosis of CRC. Notably, single phototherapy has several limitations in vivo, especially for deep tumors. Here, we discuss the advantages of the combination therapy of PDT and PTT compared with the single therapy. At the same time, this review summarizes the clinical application of PS in CRC. Although a variety of nanomaterials are in the research and development stage, few of them are actually on the market, they will show great advantages in the treatment of CRC in the near future.
\end{abstract}

Key words: colorectal cancer, photosensitizer, photothermal therapy, photodynamic therapy, nanoparticle.

\section{Introduction}

CRC is the world's third-largest cancer in men (10.9\% of the total incidence), the second-largest in women $(9.5 \%$ of the total incidence), and the second leading cause of death due to oncological causes [1]. $\mathrm{CRC}$ is the major burden to human health, due to late diagnosis and ineffective treatment approaches. Several methods are used for treatment of CRC, such as chemotherapy, surgical resection and other traditional treatments. Adriamycin is one of the most effective anti-tumor drugs used for treatment of a variety of tumors [2]. In addition, some compounds can inhibit the proliferation of CRC by reducing the content of cysteine and glutathione, thereby inducing the accumulation of reactive oxygen species (ROS) and ferroptosis [3]. Currently, surgical excision is the conventional treatment approach for CRC [4], surgery 
method is highly effective for early stage CRC. However, CRC is poorly diagnosed at early stages and early symptoms are easily confused with symptoms for other diseases. Therefore, most of CRC patients are diagnosed during advanced or metastatic stages [5]. Notably, tumors in most advanced CRC patients cannot be resected and often resistant to conventional chemotherapy. Most of CRC patients relapse after surgery and adjuvant chemotherapy during early disease stages [6, 7]. In addition, chemotherapeutic drugs are associated with toxicity to healthy tissues due to poor specificity [8]. Furthermore, these drugs have side effects, which are associated with poor specificity [9]. Therefore, studies should explore novel and effective approaches for CRC treatment. More and more researches are devoted to the diagnosis and treatment of CRC at the cellular, subcellular and molecular levels. Recently, treatment of CRC using phototherapy was reported. Phototherapy is as old as the advent of human medicine. Application of phototherapy includes killing of harmful microorganisms by sun exposure and ablation of diseased tissue using highly focused lasers [9]. Phototherapy involves interaction between light, PS and oxygen, and PS is the key to the treatment of CRC with phototherapy [8]. PDT and PTT are the main phototherapy approaches, and this article will focus on these methods.

PSs and appropriate illumination are the necessary conditions for PDT to exert tumor inhibitory effect. PDT effect is based on the concentration difference of PSs accumulated between tumor tissue and normal tissue. Irradiation using a certain wavelength of laser, changes PS energy from the ground state to a singlet excited state. The singlet excited state can be maintained for a long time through high oxidation. In the process of transition to the ground state, a large amount of singlet oxygen $\left({ }^{1} \mathrm{O}_{2}\right)$ is accumulated in tumor tissue to induce apoptosis of tumor cells through what is usually thought of as Type II reaction (In general, upon excitation, the PS is pumped from the ground singlet state to an excited singlet state, then transformed into a relatively long-lived triplet state by intersystem crossing (ISC). Then it occurs energy transfer between the triplet state of the PS and an adjacent oxygen molecule, forming singlet oxygen [10-12].). In addition, energy conversion transfers electrons to biological molecules, water, oxygen and other substances, resulting in gradual accumulation of reactive molecules species in the tumor site. Although PDT is at the experimental stage, current findings show that it is effective for diagnosis and treatment of CRC [13].
The principle behind PTT is that after accumulation of PS in the tumor site, the energy of the specific wavelength laser is converted into heat energy, causing the thermal ablation of tumor cells. Compared with the traditional treatment, PTT has obvious advantages in high uniqueness, low invasiveness, reducing normal tissue toxicity and high drug metabolism. Notably, PTT is an effective treatment approach option for CRC [14] like PDT.

PDT and PTT are modern cancer treatment methods, and their basis of action is to act on the local or systemic photosensitive compounds, known as PSs. Photosensitive molecules absorb appropriate wavelength resulting in activation of these molecules, causing cell death ultimately [15]. The characteristics of ideal PSs including: 1) high chemical purity; 2) relative stability at room temperature; 3 ) absorbance between 600 and $1000 \mathrm{~nm}$, and photosensitive effect can only be produced at specific wavelength; 4) low toxicity to other tissues and cells, and easy dissolution in human tissues; 5) high selectivity to tumor tissues; and 6) cheap, simple synthesis and easy to obtain.

The selection and application of PS is basis of light therapy, studies on PS experienced a long process, and currently only a handful of PS to get official approval. Discovery of first-generation PSs was mainly motivated by a study by Munich ascar Raab on acridine dye. Protozoa treated with dye and subjected to radiation, resulting in oxygen consumption and toxic effect, eventually died, a phenomenon known as "photodynamic effect" [16]. A water-soluble porphyrin mixture known as "hematoporphyrin derivative" (HPD) was discovered in 1970s as the first-generation PSs [15]. However, PSs have low chemical purity and poor tissue permeability. Second-generation PSs include HPDs and some synthetic PSs, such as 5-aminolevulinic acid hydrochloride (5-ALA), benzoporphyrin derivatives, and phthalocyanine [17, 18]. Compared with first-generation, second-generation PSs have many advantages, such as fewer side effects, higher chemical purity, higher ${ }^{1} \mathrm{O}_{2}$ generation rate, and stronger permeability to deep tissues. However, second-generation PSs still have the limitation of poor water solubility [15]. Development of third-generation PSs is aimed at achieving high affinity for tumor tissues to reduce toxicity on surrounding healthy tissues [15]. Studies have explored several methods to improve the selectivity of drugs, such as using tumor surface markers to combine PS with low-density lipoprotein. Recently, nanotechnology has developed rapidly, and combination and application of surface PSs and nanoparticles improves efficiency of PDT and PTT approaches. PSs can be transformed into nanoparticle 
form to further improve application and the effectiveness against tumor cells or animal models.

Nanoparticles are used as drug carriers for photosensitive molecules in optical therapy, ensuring new functions and improving their effectiveness [19]. For example; molecules with greater tissue penetration, can be used for PS delivery in PDT, whereas molecules with stronger light absorption, can be used as enhancers in PTT [20]. Several nanoparticles for application in phototherapy have been developed and are currently used in clinical research. For instance: 1) Inorganic nanoparticles used in phototherapy include metal nanoparticles (including gold nanorods [21], gold nanocapsules, gold nanocages [22], and gold nanostars [23]) whereas, carbon-based nanoparticles include fullerenes, carbon nanotubes, and graphene. In addition, other inorganic nanoparticles are used. 2) Organic nanoparticles for phototherapy include near-infrared (NIR) dyes, including indocyanine green (ICG) [24] ( a drug approved by Food and Drug Administration (FDA) for clinical use), conjugated polymer nanoparticles, and other organic nanomaterials, including phospholipid coated porphyrin. 3) Upconversion nanoparticles have stronger tissue penetration ability compared with the previously mentioned materials [25].

In this review, we introduce the development of PS, the main methods for improvement of PS, main PSs used in PDT/PTT and advances in clinical development and selectivity of PSs. In addition, we summarize the application of PDT and PTT in CRC treatment and explore their mechanisms of action. Further, we explore the effects of PDT and PTT alone and in coordination and their respective advantages and disadvantages. Moreover, we review research and development of several nanotechnologies and nanoparticles relevant to phototherapy for CRC treatment. Furthermore, application of PDT, PTT and deep tissue imaging in tumor tissues and the application of phototherapy combined with other therapies for CRC treatment are introduced.

\section{PSs and improved methods}

\section{The process of PSs induced phototherapy and the characteristics of PSs}

Light trigger therapy directly destroys cancer cells, and induces post-treatment effects. It improves efficacy of combined therapy by enhancing intratumoral penetration and entry of nanoparticles into cells, accelerating drug release and improving immune response to cancer cells[26]. PDT kills cancer cells and improves survival and quality of life in cancer patients. Several new approaches have been developed to improve efficacy of PDT[27]. PS is irradiated to produce ROS or monomorphic oxygen that acts on cancer cells and causes irreversible damage to cancer cells through photo action [28]. Targets for photo action include epidermal growth factor receptor, cholesterol and low-density lipoprotein, estrogen receptor, nucleus and DNA, folic acid receptor, cholecystokinin receptor, lectin carbohydrate receptor and tumor-specific antibody [29]. Halogen lamps, arc lamps, lasers and light-emitting diodes are used to activate PS, however they are all visible light with high tissue absorption, leading to low tissue penetration thus have low treatment efficacy. NIR region wavelength ranges from 600 to $1000 \mathrm{~nm}$ and can minimize tissue attenuation to maximize effectiveness by ensuring tissue penetration [9]. Long wavelength light activates PS and kills cancer cells concurrently, however, it causes irreversible damage to normal cells. Therefore, excitation wavelengths of PS used in treatment of CRC is mainly concentrated in the NIR region. PSs produce heat just like light action to kill cancer cells under different wavelengths of light in a process-known as photothermal action. In addition, when tumor is heated to $42^{\circ} \mathrm{C}$ by photothermal action, the cancer cells are more susceptible to other treatments [26]. Photosensitive therapy has unique advantages such as spatiotemporal selectivity, high efficiency, no drug resistance and non-invasiveness which are not present in conventional approaches. Excitation wavelength of PS in the NIR has the advantages of NIR light activation, low invasive treatment, non-specific activation, high temporal and spatial accuracy, and real-time dose adjustment. PSs can be grouped into various types based on their carriers, including inorganic PSs, organic PSs and metal PSs [26]. Although PSs can kill cancer cells, preclinical and clinical studies report a few shortcomings, which hinder their development. Therefore, PSs should be modified to reduce its limitations and improve effectiveness.

\section{Improvement of PS}

\section{Increasing penetration depth}

Different wavelengths of light have different ability to penetrate tissue. Longer wavelength of light show, stronger ability to penetrate tissue compared with short wavelength light. For instance, the activation wavelength of photofrin is $630 \mathrm{~nm}$, however, its penetration depth is only about $0.5 \mathrm{~cm}$, therefore, it is not ideal for large or deep tumors. On the other hand, with wavelength of the light source approximately $800 \mathrm{~nm}$, results in tissue penetration depth of $1 \mathrm{~cm}$. However, short-wavelength light sources have higher ${ }^{1} \mathrm{O}_{2}$ yield and shorter irradiation 
time compared with long-wavelength sources [30]. One of the solutions to improve the penetration of deep tissue lesions is to use the long wave near-infrared (NIR) light in the range of $600 \sim 1000$ $\mathrm{nm}$, known as the "NIR window" or "optical window" [31]. NIR light is preferred [32]. Therefore, current studies are exploring the best irradiation wavelength with high ${ }^{1} \mathrm{O}_{2}$ yield. Studies report that femtosecond lasers are used to drill holes in tumor tissue in advance to enhance tissue permeability of PS, thus improving its killing depth. However, studies have not explored the effect of this method on spreading of tumors [30].

\section{Enhanced targeting}

Tumor cells require higher glucose levels compared with normal cells (a phenomenon known as the Warburg effect [33]) and this effect can be extended to a variety of other carbohydrates, including mannitose and galactose [34]. Therefore, recent studies have exported conjugation of PSs with sugars to increase selective recognition or uptake by cancer cells. Nanomedicine and nanoparticle therapy have several advantages compared with traditional therapies, however their specificity is low, and these methods have poor pharmacokinetics. Nanoparticles have attracted extensive research attention due to the variety of potential modification [35]. Structure of nanoparticles can be used as a multifunctional assembly platform [36-38], enabling combination of chemotherapy drugs and cancer imaging agents [39]. The structure ensures high solubility and colloidal properties, which can be used in complex environments (such as blood and tissue)[28]. Gold nanoparticles, for example, have good biocompatibility and can accumulate passively within tumors by enhancing permeability and retention. Moreover, gold nanoparticles are efficient at converting light energy into heat energy [39].

\section{Improvement of safety}

One of the adverse effects of PDT is the 'always-on' phenomenon, which requires patients to be kept in the dark for a long time (usually several weeks) after treatment. An adaptive PS should be developed that can be activated in the tumor microenvironment to produce ROS to avoid low tumor specificity of PS and the 'always-on' phenomenon. Supramolecular switches were designed and synthesized based on a host guest complex containing water-soluble column aromatic hydrocarbon (WP5) and AIEgen PS (G) [40]. Formation of the host-guest complex WP5, quenches fluorescence and inhibits generation of $G$ ROS. Binding sites between $G$ and WP5 change through shuttle motion in acidic environments due to the $\mathrm{pH}$ responsiveness of WP5. Therefore, fluorescence and ROS production of host-guest complexes can be turned on at $\mathrm{pH}$ 5.0. Tumors thrive in acidic environments; therefore, this switch with good $\mathrm{pH}$ responsiveness and stability can be used in cancer therapy.

\section{Stimuli-responsive PS activation}

When the nanoparticles loaded with Ps reach the tumor tissue, the Ps can be activated by the stimulation of tumor microenvironment. In this case, the main factors of activating $\mathrm{Ps}$ are: $\mathrm{pH}, \mathrm{H}_{2} \mathrm{O}_{2}$, adenosine triphosphate (ATP), and temperature. [PHC] PP@HA was assembled from PDA, CE6 and hemoglobin in the ratio of 1:2:4. Compared with normal tissue, the $\mathrm{pH}$ value of tumor tissue was lower. When the $\mathrm{pH}$ value was normal, the compound aggregated and decomposed into single up transformed nanoparticles in the endometrium / lysosome of tumor cells. At the same time, polychloride e6(Ce6) decomposes into an extinguished state, and PS can be activated effectively [41]. Due to rapid growth and insufficient blood supply, tumor tissue is prone to hypoxia. Based on the difference between podophyllotoxin and normal tissues, a novel podophyllotoxin prodrug (POD-PEG) can be synthesized by combining podophyllotoxin with oxalate bond of poly (ethylene glycol)(n) mono methacrylate, which can self-assemble into stable nanoparticles. Oxalate bond can react with endogenous $\mathrm{H}_{2} \mathrm{O}_{2}$ in tumor to produce $\mathrm{O}_{2}$, which can alleviate tumor hypoxia and provide more molecular oxygen for PDT when nanoparticles are degraded and activated [42]. It has been confirmed that the concentration of ATP in tumor tissue $(>100 \mu \mathrm{m})$ is higher than that in normal tissue $(1-10 \mathrm{~nm})$, which provides the possibility for ATP to selectively activate PSs. Gao et al. first synthesized a macrocyclic amphiphile based on guanidinium-modified calix [5] arene pentadodecyl ether (GC5A-12C), which co-assembled with 4-(dodecyl oxy) benzamidoterminated methoxy poly(ethylene glycol) (PEG-12C) and PSs to form NPs. Nanoparticles accumulate in tumor tissue through EPR effect and are replaced by ATP, which is released with the recovery of fluorescence and photoactivity [43]. Hydrogel is thermo responsive self-healing. After adding polydopamine nanoparticles (PDA NPs), the SP-(DMAEMA-co-HEMA-AA)/PEI/PDA-NP

nanocomposite hydrogel presented phase change and volume shrinkage under NIR irradiation. The effect of photothermal effect of PDA NPs on the temperature of hydrogel in the corresponding region is increased by near infrared laser irradiation. Because of the 
hydrophobicity-hydrophobicity transition of hydrogels, DOX molecules with antitumor activity are extruded from hydrogels at temperatures above their lower critical solution temperature (LCST), thus the tumor cells are inhibited by the internal stress of the shrinking hydrogels [44, 45].

\section{Targets of PSs}

PSs for CRC can achieve the requirements of precision medicine through multiple levels of targets. At present, the targets for PSs diagnosis and treatment are mainly divided into three types: cell, organelle and molecular targets.

\section{Cellular level}

PSs regulate autophagy of cells, specifically by acting on target cells, affecting specific targets in cells, and inducing apoptosis or necrosis of target cells [46]. Cancer stem cells are the most ideal target, and PSs can play a role before or in early stages of disease effectively. Jun Ki Kim et al. reported that GFP-Lgr5 + stem cells located on the surface of the colon cavity are near the center of CRC. Targeted therapy for this cell type has potential preventive effect on tumor growth, especially for high-risk population of CRC [47].

\section{Organelle level}

Nano drugs have the ability to kill tumor cells by initially targeting organelles, resulting in a series of subsequent reactions [48]. By targeting specific receptors of a specific organelle, corresponding signaling network is activated, then the protein expression and activation level of the signaling pathway are up-regulated or down-regulated. Further, the cell death pathway can be initiated to achieve the purpose of cancer treatment by killing cancer cells. Numerous researchers have devoted themselves to the research and development of drugs targeting the organelles of CRC based on this mechanism. Kaiyuan $\mathrm{Ni}$ et al. reported a mitochondrion targeted metal organic frameworks (nMOFs) named Hf-DBB-Ru, which induces the apoptosis of tumor cells in mouse model of CRC by depolarizing the mitochondrial membrane[49] (Figure 1). Sharmin akter et al. explored the lysosomal targeted talaporfin sodium loaded with inactivated virus particles-Laserphyrin ${ }^{\circ}-\mathrm{HVJ}-\mathrm{E}$ (L-HVJ-E), which induces apoptosis and necrosis of human prostate cancer cell line PC-3, depending on light dose and concentration[50]. Modified iridium (III) protein complexes have excellent performance of aggregation in endoplasmic reticulum at low concentration [51]. Most of the targeted PSs have good localization ability in organelles, but only a few can reach the nucleus. Wen Pang et al. reported that the effective carbon point (C point) with intrinsic nucleolar targeting ability can be used as PS to improve therapeutic performance under low dose PS and light irradiation [52].

\section{Molecular level}

Advancements in molecular biology technology have resulted in development of application prospects for prevention, diagnosis and treatment of CRC by targeting specific molecules or molecules different from normal tissue expression. These molecules include almost all kinds of substances molecules in the whole entire process of protein expression, such as genes, RNA and proteins. Here, in this review, we discuss common molecular targets for CRC.

\section{Genetic targets}

TP53 Inactivation of TP53 is one of the common causes of cancer, and the inactivation of tyrosine kinase can cause the inactivation of TP53 through the Wnt signaling pathway, which will further enhance the invasion ability of CRC cells. Deterioration of CRC can be slowed down by increasing the expression efficiency of the two genes [53].
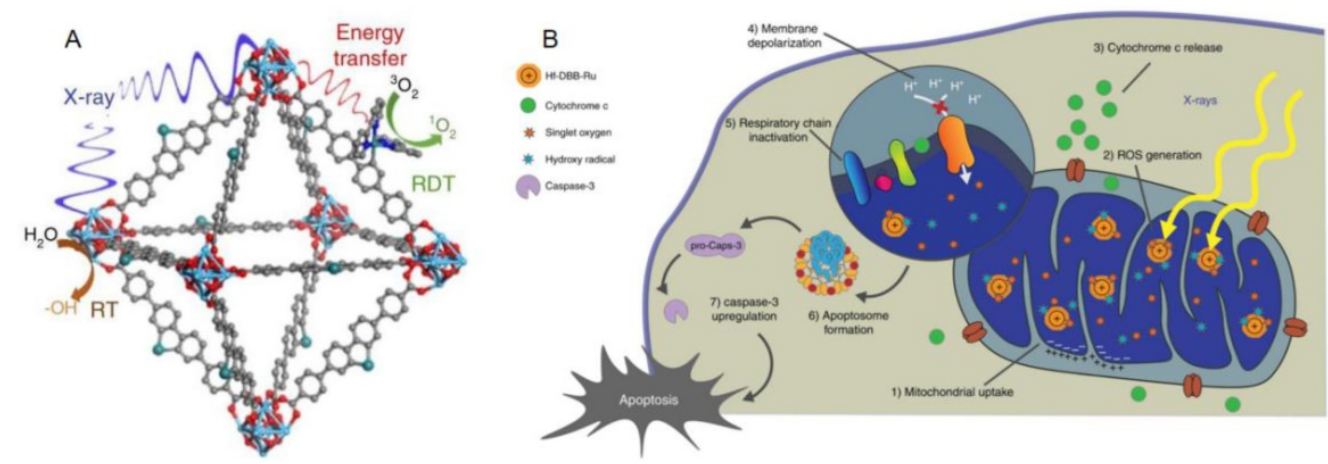

Figure 1. Structure and Function mechanism of nMOF. (A) Schematic showing the RT and RDT process enabled by Hf-DBB-Ru.6 (B) The process of Mitochondria-targeted RT-RDT mediated by Hf-DBB-Ru [49]. 


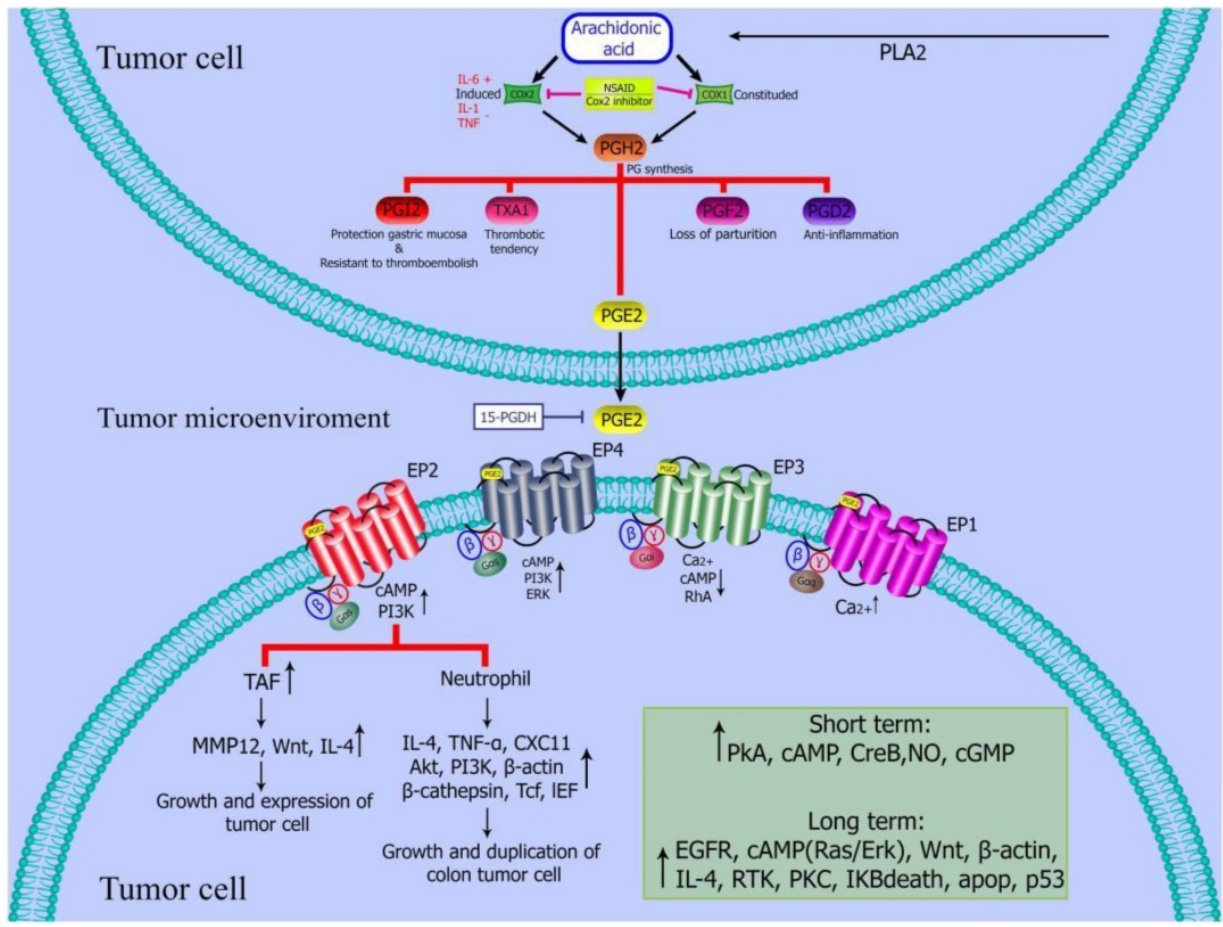

Figure 2. PGE2 regulates tumor microenvironment. PGE2 inhibits the activation of dendritic cells and $B$ cells, and induce the activation or formation of $M 2$ polarized macrophages, myeloid suppressor cells (MDSCs) tumor associated fibroblasts and mast cells [55].

BRAF

The Ras / Raf / MEK / ERK signaling cascade is known as mitogen activated protein kinase (MAPK) pathway, and BRAF is a representative member of the RAF family. Abnormal activation of this pathway may lead to mutation of the BRAF gene, thus inhibiting its antitumor effect. Upregulation of the expression of BRAF gene by targeting is a potential target for the treatment of CRC [54].

\section{Protein targets}

\section{PEG2}

Colon cancer can be induced by the increase in the prostaglandin level, especially prostaglandin E2 (PEG2). Targeting COX-2 / PGE2 / EP receptors may be considered as an effective treatment strategy for colon cancer. For example, NSAIDs can reduce the expression of PGE2 by administration of enzymes that inhibit Cox to reduce CRC [55] (Figure 2).

\section{AEBPI}

Overexpression of adipocyte enhancer binding protein (AEBP1), a transcriptional repressor, is correlated with the occurrence and development of tumors. Therefore, this protein can be used as a molecular marker for targeted therapy [56].

\section{$\mathrm{Bcl}-2$}

Bcl-2 is an anti-apoptotic protein that is closely associated with the occurrence of several cancers. BH3 mimics can specifically inhibit the expression of Bcl-2, and change the imbalance of apoptosis of CRC cells [57] (Figure 3).

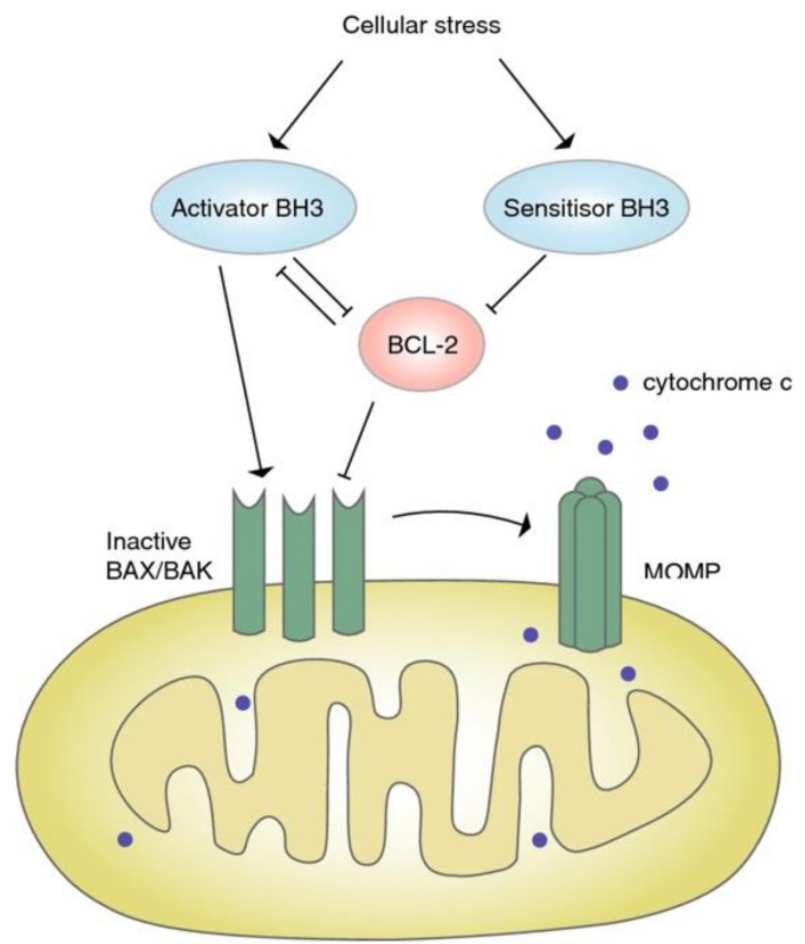

Figure 3. Signaling pathway of BCL-2. Under the pressure, the expression of $\mathrm{BH} 3$ increased, which further blocked the expression of $\mathrm{Bcl}-2$, inactivated Bax/Bak, and resulted in the closure of MOMP and apoptosis [57] 


\section{PD-1/PD-L1}

Checkpoint block is one of the important mechanisms of tumor immunotherapy. Drugs targeting PD-1/PD-L1 checkpoint shows a relatively long-term tumor growth inhibition. The combination of photosensitive material and related monoclonal antibody shows significant inhibitory effect on CRC cells [58].

\section{EGFR}

Given that CRC is easy to metastasize, the strategy of inhibiting malignant transformation is more reliable. Inactivation of EGFR or blocking of the EGFR-related pathway is an effective approach for diagnosis and treatment of CRC [59] (Figure 4). Cetuximab and Panitumumab have been widely used for the treatment of metastatic CRC. Cetuximab is a chimeric (IgG1) monoclonal antibody (mAb). It blocks the binding of endogenous ligands by binding to the extracellular domain of EGFR, which significantly inhibits the growth of tumor cells. In addition, it may also have immune-mediated antitumor effects, such as antibody dependent cell-mediated cytotoxicity [60].

\section{Photodynamic therapy}

\section{Targeted transport process and mechanism of PS}

Several recent studies explored the mechanism of PDT at the cellular and molecular level. The basic process of PDT is that PS is injected into the body, and is accumulated in tumor tissues or cells through passive or active targeting and the lesion is irradiated with specific wavelengths of light. Irradiation then stimulates PSs to produce ROS, leading to tumor cell death (through apoptosis, necrosis, and excessive autophagy processes [61]). Accumulation of PS in tumor cells or tissues is achieved by passive or active targeting in vivo. Passive targeting refers to specific accumulation of PS in tumor tissues due to the difference in physiological conditions between tumor tissues and normal tissues after entry of PS into the human body. The differences between tumor cells and normal tissue cells are as follows: 1 . Metabolism of tumor tissue is more vigorous compared with that of normal tissue, and $\mathrm{pH}$ is lower compared with that of normal tissue; 2. Tumor tissue contains more lipoprotein; 3. The space between tumor tissue and normal tissue is larger; 4 . Tumor tissues are characterized by more macrophages and poor lymphatic circulation. Active targeting is the process of combining PS with tumor cell proliferation, metabolism, vascular, and other specific factors (such as tumor antigen, serum protein and epidermal growth factor [62]) to achieve PS specific recognition of tumor tissue. PS nanoparticle leles, light, and ${ }^{1} \mathrm{O}_{2}$ are the three important factors of PDT. PDT achieves synergistic effect through combination with traditional therapy and has a very broad application prospect in clinical treatment of tumor.

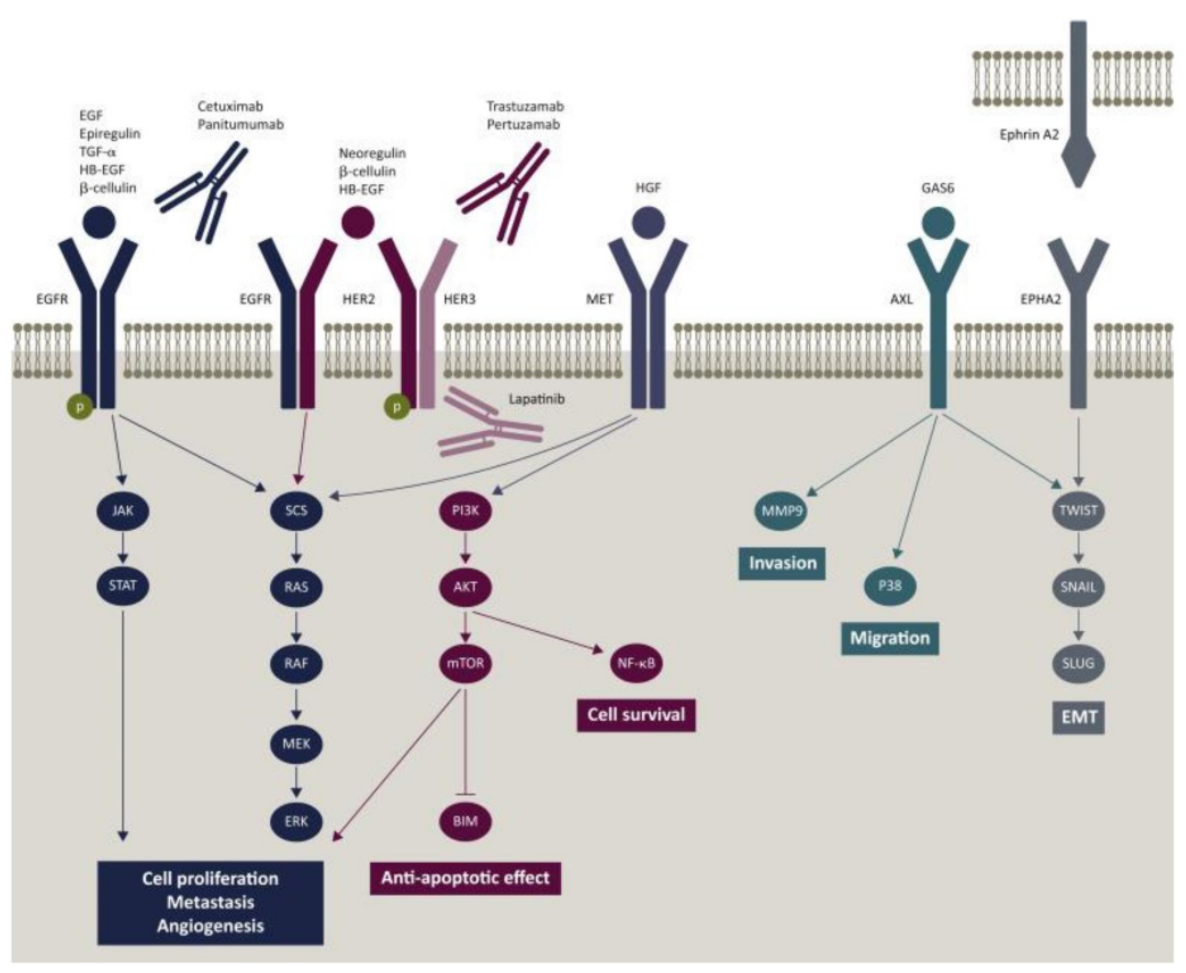

Figure 4. Epidermal growth factor receptor (EGFR) signaling pathway and potential mechanism of resistance to cetuximab and panitumumab [59]. 


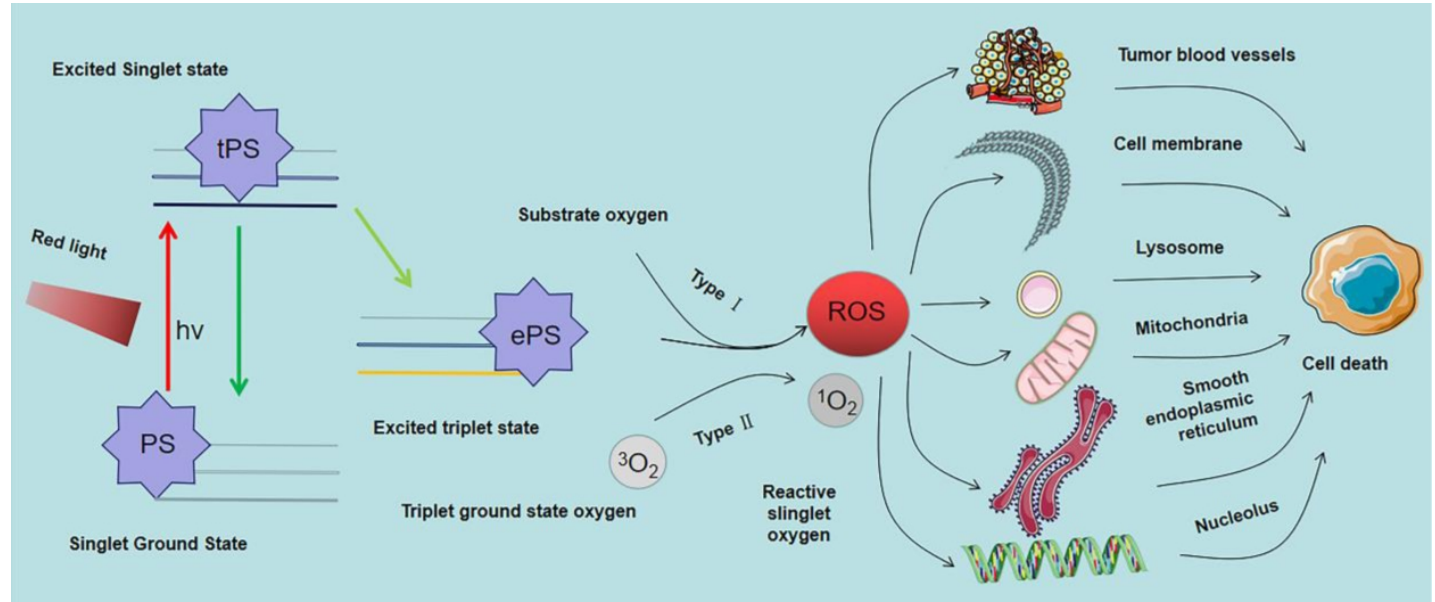

Figure 5. Mechanism of photodynamic reaction and targets of ROS singlet ground state. The Ps transits from singlet ground state to triplet ground state and produces ROS through type I reaction, and ROS can mediate tumor cell death by acting on various organelles and tumor microvessels.

\section{Photodynamic molecular reaction of PS}

After entry of the PS into the cell or tissue it is absorbed by light of specific wavelength. The absorbed energy changes the PS from ground state to singlet excited state, and then to triplet excited state through intercellular channeling. The triplet excited state reacts with the substrate to produce ${ }^{1} \mathrm{O}_{2}$ and ROS. These products are cytotoxic; therefore, they kill tumor cells directly or indirectly. This process is mediated by the light in the PDT reaction that can be generally divided into type I and type II reactions. However most PDT reactions are type II reactions [63]. As shown in Figure 5, In type I reactions, triple excited PS nanoparticles react with cell membrane or biological macromolecules to transfer a hydrogen atom or electron to form free radicals. These free radicals then interact with oxygen to generate ROS (such as superoxide anion, hydroxyl radical and hydrogen peroxide) to kill targeted cells. In type II reactions, triple laser PS directly transfers energy to oxygen molecules to generate free radicals. ${ }^{1} \mathrm{O}_{2}$ (also a kind of ROS, different from type I reaction products) has high cytotoxic effects, and is a highly reactive substance that reacts with oxygen-sensitive groups in biomolecules (such as unsaturated fatty acids, proteins and nucleic acids) to cause oxidative inactivation and significant damage to tumor cells. In contrast to other ROS, the ${ }^{1} \mathrm{O}_{2}$ lifetime is low, approximately $10-320 \mathrm{~ns}$ the diffusion and migration ability in cells and tissues is weak, approximately 10-55 nm, which limits its action range [64]. These findings imply that the location of activated PS determines the area of PDT causing direct tissue damage [65].

\section{Damage mechanism induced by PDT}

Studies report that the mechanism of action of
PDT at the molecular level indicates is through PSs aggregating in tumor cells under the action of relevant light sources. After aggregation, ${ }^{1} \mathrm{O}_{2}$ and ROS are produced, thereby destroying cell structure, causing oxidative damage to cells, and ultimately leading to cell death [66]. At the subcellular level, PSs selectively accumulate in cell membranes, mitochondria, lysosomes, and other subcellular structures, thus directly damaging these cell structures. Damage of organelles to a certain degree results in cell death. In addition, these activities induce programmed cell death. Different types of PSs have different preferential accumulation sites, and the same PS can cause orderly or simultaneous damage to multiple organelles.

\section{Mitochondrial injury}

Mitochondria are the most vital organelles in production of cellular energy. Mitochondria host a series of vital life activities such as cellular aerobic respiration and tricarboxylic acid cycle. Several types of PSs target mitochondria. Fluorescence microscopy shows that the second-generation PS, 5-ALA is mainly distributed in mitochondria, and its mechanism of action is by changing mitochondrial membrane potential. Some mitochondria are damaged after 5-ALA therapy, cristae of mitochondrion become invisible, and vacuoles and swelling are observed. ROS produced by PS decreases mitochondrial membrane potential and resulting in depolarization, causes disappearance of mitochondrial cristae, and expansion leading to rupture of the outer membrane. Destruction of mitochondrial structure interrupts respiratory activity and oxidative phosphorylation and inhibits calcium uptake. In addition, apoptotic effectors in the mitochondrial intermembrane space are released to induce apoptosis. For example, cytochrome $\mathrm{C}$, apoptosis-inducing factor and calcium 
ion ${ }^{[67]}$ are released into the cytoplasm, acting on calcium-dependent proteins or cause destruction of cells by activating apoptotic signals such as caspases, $\mathrm{Bcl}_{2}$ and Bax, etc. Chromatin in the nucleus is destroyed, resulting in cell dysfunction, generation of apoptotic bodies, and apoptosis [68] (Figure 6).

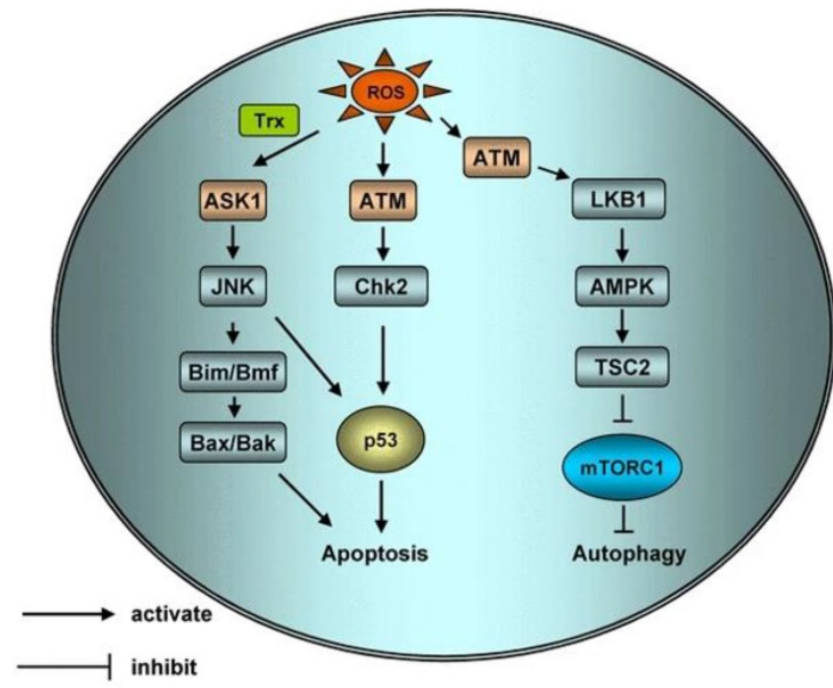

Figure 6. Schematic of apoptotic and autophagic signaling upon ROS. ROS can activate Apoptosis Signal Regulating Kinase 1 (ASK1) by activating sulfur peroxide protein, ASK1 can regulate protein phosphorylation of JNK, ASK1 can activate Bax and Bak or increase the expression of $\mathrm{p} 53$ through Bim and BMF to initiate apoptosis. $\mathrm{H}_{2} \mathrm{O}_{2}$ activated ATM independently by MRN/ser-1981. Activated ATM induces apoptosis and autophagy through CHK2/p53 and LKB1/AMPK/ TSC2/ mTORC1 pathways respectively [69].

\section{Lysosomal damage}

PSs targeting lysosomes mainly cause cell death through apoptosis. Photodynamic action triggers primary damage of lysosomes, activates acid sphingomyelinase, and releases ceramide, leading to cell apoptosis [70]. The $\mathrm{pH}$ of lysosomes in tumor tissues is lower compared with that in normal cells, thus it stimulates $\mathrm{pH}$-activated PS. Therefore, after PSs are transported into lysosomes, they are activated, causing lysosomal membrane damage ultimately inducing apoptosis [71]. Furthermore, lysosomal damage can also indirectly trigger mitochondrial apoptosis by transferring some apoptotic proteins between organelles.

\section{Cell membrane damage}

Damage mechanism of PS targeting cell membranes is through inducing apoptosis, Initiation of apoptosis occurs through two pathways. The extracellular pathway refers to the combination of PSs with tumor necrosis factor gene family death receptors on the cell membrane and membrane surface. This combination activates transforming protein, activates caspase- 8 apoptotic protein, and cascade reaction leading to apoptosis. In the internal pathway PS induces apoptosis by recognizing receptors on the surface of the cell membrane, enters cells, and binds to the mitochondria and lysosomes, etc. through the internal pathway of organelles [72].

\section{Nuclear and genetic material damage}

PSs, such as phenocyanines and HPD, bind to the nuclear membrane after enter into cells. PSs get to hinder cytoplasmic separation and cause significant damage to chromatin, leading to production of multinucleated cells and malformed nuclei. Chromosomes and chromatin are implicated in different stages of cell mitosis and cells in different stages have varied sensitivity to PS. S and G1 phases are more sensitive to PS, whereas the M phase is less sensitive to PS. PS attached to the nuclear membrane can directly damage the nucleus and DNA during mitotic metaphase [73]. Analysis of the ultrastructure shows that guanine in DNA is deformed, leading to changes in DNA single-strand breaks, chromosome aberrations, sister chromosome exchanges and DNA-protein crosslinks, etc [74].

\section{Damage to blood vessels in tumor tissues}

Tumor vascular tissues have different physiological characteristics compared with normal tissues, such as abnormal endothelial morphology, discontinuity, irregular shape, enlarged lumen, increased leakage, partial loss of adventitia and basement membrane, and tumor cell embedding [75]. ${ }^{1} \mathrm{O}_{2}$ in the photodynamic action of PS causes acute damage to microvessels at the tumor site, resulting in vascular obstruction, which in turn causes cell necrosis due to inadequate access of tumor cells to oxygen [76].

\section{Involvement of the immune system}

Immune system PDT induces local tissue edema, which is a manifestation of immune system involvement in regulation. Korbelik et al. [77] treated normal BALB/c mice and immune deficient mice with PDT and reported that recurrence rate of tumors is higher in immune deficient mice. This finding indicates that the body requires an immune response to clear remaining cancer cells after treatment. PDT promotes release of immune inflammatory factors, stimulates and induces immune response. Gollnick et al. [78] report that tumor cells after PDT treatment have immunogenicity and can stimulate the specific immune response. Combination of PDT and immunotherapy-photodynamic immunotherapy high potential for treatment and control of malignant tumors as well as in prevention of tumor metastasis and recurrence. 


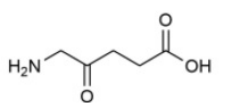

5-ALA

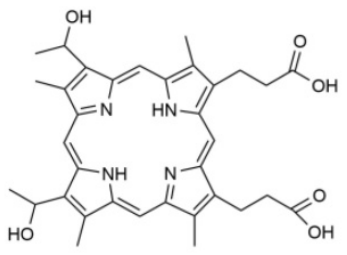

Hematoporphyrin
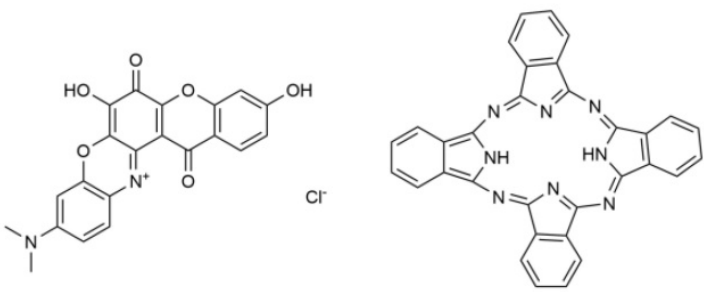

Phenocyanine

Phthalocyanine

Figure 7. Molecular structure formula of some PSs.

\section{Application of classical PS in treatment of CRC}

Since the discovery of the photodynamic effect of some dyes and crude hematoxylin in 1920, advances in development of PSs have been achieved resulting in three generations of PSs. PSs are modified by combination with tumor cell targeting specific groups and carriers, such as monoclonal antibodies, polypeptides, somatostatin, folic acid and LDL, etc. In 1993, hematoporphyrin as shown in Figure 7 was first used to treat bladder cancer in Canada and was further used for treatment of the esophagus, lung, stomach, and cervix tumors [79].

HPDs have high efficacy; however, studies report that HPDs have severe side effects. HPDs accumulate in the skin after entering the body of patients, and the clearance of drugs from the skin is slow. Therefore, patients need to be protected from light for 4-6 weeks after using drugs. These side effects greatly limit the clinical application of HPDs.

5-ALA is a PS precursor with high activity, high fluorescence intensity, convenient administration, safety, and skin allergic reaction, which can generally be eliminated within $48 \mathrm{~h}$ [80]. A study by Luo Li ping, et al. 120 CRC grouped patients into two groups to undergo conventional chemotherapy. The chemotherapy regimen was PF regimen [fluorouracil and cisplatin][66], where patients were subjected to 12 cycles of chemotherapy, and underwent PDT treatment one week after chemotherapy. Patients in the control group were treated with HPD injection as the PS approach, whereas the PS in the observation group was 5-ALA injection. The two groups were treated separately. The experimental results showed that the survival time and rate of the observation group were significantly higher than compared with those of the control group, and 5-ALA had a significant role in treatment of CRC.

\section{Photothermal therapy}

\section{Mechanism of PTT}

Photothermal therapy is a treatment method that uses materials with high photothermal conversion efficiency to inject them into the human body. The approach used targeted recognition technology to gather near the tumor tissue, and converts the light energy into heat energy under irradiation of NIR light to kill the tumor. Tumor cells have lower heat tolerance compared with normal tissue cells, therefore the balance has less impact on normal cells [81].

\section{PTT injury mechanism}

\section{Direct cell damage}

After the ablative agent enters the tumor cell, it generates heat under irradiation of NIR increasing the temperature of the tumor cell. High temperatures change permeability of the cell membrane, affect the sodium-potassium pump and protein ion channel, and affect selective permeability of the cell. In addition, this phenomenon causes destruction of cell membrane structure, disorganizes the cytoskeleton, impairs cell function, and leads to cell death. Furthermore, high temperatures can change structure of chromosomes and inhibit their synthesis, thus affecting DNA repair and causing damage to genetic material [82].

\section{Damage to blood vessels in tumor tissue}

Tumor tissues and normal cell tissues have significant differences in physiological morphology. Tumor tissues are characterized by an abnormal growth of blood vessels, structural disorders, sinusoid formation when capillaries are compressed low heat response and large blood flow. Generation of heat by the ablative agent results in low blood flow inside the tumor tissue, increases resistance, lowers heat dissipation levels, and significantly increases temperature, resulting in insufficient cell oxygen and $\mathrm{pH}$ value lower than normal, ultimately inducing cell apoptosis[83], blood vessels of normal tissues expand under high temperature, blood flow is accelerated, blood circulation is good, and heat dissipation is rapid. PTT affects repair of tumor vascular by inhibiting tumor-derived vascular endothelial growth factor and its expression. These changes hinder endothelial cell proliferation and inhibit tumor growth and metastasis. 


\section{Types of ablation agent}

Ablative agents for PTT use should have good biocompatibility, good photothermal conversion efficiency, and good biological targeting. Nanoparticles have selective photothermal conversion efficiency and can passively accumulate in tumor cells [84]. Nano drugs have several advantages in targeting tumors in tumor treatment, therefore, nanoparticles are widely used as ablators in PTT. Nanomaterial ablators for PTT can be divided into precious metal nanoparticles, semiconductor nanoparticles, carbon-based nanoparticles, and organic nanoparticles based on the material used for their development [14].

\section{Noble metal nanoparticles}

Surface plasmon resonance (SPR) of noble metal nanomaterials is the basis for their photothermal conversion ability. Absorption wavelength and photothermal conversion efficiency of metal nanoparticles depend on the SPR effect and are adjusted according to the size and structure of these nanoparticles [85]. In 2017, Hosseinzadeh et al. used gold nanosphere particles, which had a maximum absorption wavelength of $630 \mathrm{~nm}$ and was in the visible wavelength range to target MUC1 aptamer in vitro. Gold nanoparticles can reduce the active migration ability of CRC cells through photothermal effect, however, the absorption peak of visible radiation limits the application of pure gold spherical nanoparticles [86]. The absorption peak of gold nanoparticles can be adjusted by hybridizing them with other materials. Gold nanoparticles have the best PTT efficacy in the NIR region [87]. The absorption peak of gold nanorods can be adjusted to the NIR by adjusting the aspect ratio [21]. Gold nanoshells can be used for adjustment of absorption peaks to the NIR by adjusting size and thickness [88]. Gold nanoparticles have significant photothermal effects on tumor cells and tissues. However, low photostability of gold nanoparticles results in structural deformation [89], which limits application and development of gold nanoparticles. Future studies should design a gold nanomaterial structure with good photostability and high photothermal effect.

\section{Carbonyl nanoparticles}

Carbonyl nanoparticles have electrochemical and non-covalent bonding properties, good physical properties, significant absorption in the infrared region and are widely used as ablators. Development of carbonyl nanoparticles, such as graphene and carbon nanotubes [90] for use in PTT is currently underway. Graphene structure consists of flat carbon atom sheets whereas carbon nanotubes are three-dimensional structures made of graphene. The two materials can be combined with magnetic materials and chemotherapeutic drugs to act as ablators [91]. Graham EG et al. combined MWCNTs with folic acid for treatment of colon cancer cells. Combination of carbonyl nanoparticles with folic acid, increased the affinity of the structure to CRC cells increased by four-fold to five-fold, whereas activity against CRC cells decreased by $50 \%$. Markovic et al. [92] analyzed the type of 20 cell death caused by graphene-induced PTT. Cells were found to die by necrosis and apoptosis according to their characteristics. However, studies on carbonyl nanoparticles show low photothermal conversion efficiency and require higher light intensity or wavelength. Excessive intensity and wavelength of light damages normal cellular tissues, results in easy deposition into organs such as the liver, kidney, and skin, causing granuloma formation and leading to the development of cysts and organ damage [92].

\section{Semiconductor nanoparticles}

Semiconductor nanoparticles have the advantages of low cost and cytotoxicity unlike noble metal nanoparticles. Copper chalcogen nanoparticles are the main types of semiconductor nanoparticles. Photothermal conversion efficiency of copper-based nanoparticles can be improved by adjusting the size and shape. In $\mathrm{Hu}$ [93] and other experiments report synthesis of $\mathrm{Cu}$-based nanoparticles with uniform 3D floral morphology. In these studies, a hydrophilic copper substrate-like nanomaterial was developed. Its photothermal conversion efficiency was up to $25.7 \%$, which is slightly higher compared with that of gold nanomaterial under the same wavelength of irradiation. Studies report use of semiconductor nanoparticles have been used for treatment of CRCs with high effectiveness. Copper sulfate nanocomposites $\left(\mathrm{CuSO}_{4} \mathrm{NCs}\right)$ reduces viability of CRC cells after NIR irradiation [94]. Polyethene glycol-coated copper nanowires (PEGylated CuNWs) induce tumor cell necrosis and inhibit tumor growth in CRC subcutaneous tumor-bearing mice by intratumoral injection and NIR irradiation [95]. In a study by Hessl et al[96], the synthesized copper selenide nanocrystals induced tumor cell death under NIR irradiation. Shortcomings of semiconductor nanoparticles are similar to those of noble metal nanoparticles. They have low photothermal conversion efficiency and require a high intensity of light irradiation. These particles can easily cause damages to normal tissue cells.

\section{Organic nanoparticles}

Organic nanoparticles have better 
biocompatibility and biodegradability compared with inorganic nanoparticles [97]. Therefore, high numbers of organic nanoparticles are used in PTT. Organic nanoparticles under evaluation include NIR dye-based nanomicelles, porphyrin liposome nanoparticles [98], small organic nanoparticles and organic polymer nanoparticles. NIR dye-indocyanine green nanoparticles can be excreted through urine, thus reducing cytotoxicity caused by drug accumulation. In addition, nanoparticles carrying liposomes and proteins can be degraded in vivo. Furthermore, organic nanoparticles can be combined with functional molecules to prepare better nanoparticles and can effectively slow growth rate of tumor volume, causing tumor tissue necrosis. Sensitivity of photobleaching is a defect of organic photothermal agents. Absorption capacity of organic photothermal agents decreases rapidly and cannot accept long-time light source irradiation under prolonged irradiation.

\section{Application of imaging examination and PDT / PTT in tumors}

Due to the diagnosis of CRC and detection imaging methods, there is a big gap result in a lot of problems in the early diagnosis of CRC. Phototherapy, a promising method for targeted treatment of tumors, is effective in treatment of CRC. Combination of PDT and PTT with various imaging examinations of CRC ensures early treatment of patients thus reducing cases for surgery requirement [99]. Image-guided therapy can integrate various information to infer distribution and metabolism of PSs [100]. In addition, integration of imaging and treatment into single-imaging guided multifunctional cancer treatment platform improves treatment efficiency and safety of this approach [101].

"Therapeutics" refers to combination of therapeutic model and diagnostic imaging in one system, among which noninvasive phototherapy has attracted extensive attention in recent years.

\section{Combination of fluorescence imaging with PDT / PTT for tumor treatment}

In the process of high-energy fluorescence, a kind of fluorescence signal with different orbital wavelengths is produced when high-energy electrons are irradiated to ground state. Fluorescence imaging is collection and recording of the intensity of emitted light signal in a certain range. It is characterized by high spatial-temporal resolution and short imaging acquisition time. Advantages of fluorescence imaging include high stability, low toxicity and low cost. PS used in PDT and PTT have strong absorption in the NIR, which can convert the absorbed NIR light energy into fluorescence, ROS, and heat. Fluorescence imaging has the characteristics of tissue spontaneous fluorescence and light scattering in the "NIR optical window" [102]. A variety of multi-functional diagnosis and treatment systems for cancer NIR fluorescence imaging and combined phototherapy are currently available. Naphthalocyanine (NC) has a strong absorption near $800 \mathrm{~nm}$, which provides a broad application prospect for fluorescence imaging and combined phototherapy of deep tumors[103]. However, due to the planar structure of $\mathrm{NC}$ molecules, their solubility in water and aggregation are limited. Sigh et al. integrated SiNC and IR780 dyes into mesoporous silica nanoparticles and explored their photothermal ability under light irradiation and their potential for use as nanoparticles for fluorescence imaging and PTT [104].

Effective packaging strategy reduces aggregation of SiNC molecules, retains the fluorescence signal of NIR, stabilizes the characteristics of PDT and PTT, and enhances the water solubility [103]. Application of SiNC-NP in development of NIR can be evaluated by recording the strong fluorescence signal in the tumor area. Previous studies report advances in development of fluorescence imaging for PTT and PDT guidance. The methods have a spontaneous fluorescence background and limited penetration depth compared with other imaging technologies. Moreover, NIR fluorescence NPs has low quantum yield and limited fluorescence signal $[105,106]$.

\section{Combination of photoacoustic imaging with PDT / PTT for tumor treatment}

Photoacoustic imaging (PAI) is a new non-invasive and non-ionizing biomedical imaging method. Phototherapy, combined with diagnostic photo imaging, has great potential for precision tumor therapy. PAI provides tissue imaging information at a certain depth of penetration and has high image contrast and spatial resolution [107]. PAI is often combined with PTT because photoacoustic signals originate from photothermal conversion generated by the absorption and excitation of PS [20]. PAI is a combination of photoacoustic microscopy (PAM) and photoacoustic tomography (PAT)[61]. PAM and photoacoustic endoscopy can be used for diagnosis of CRC. However, due to the low imaging speed, PAT has a larger penetrating field and deeper area compared with PAM [105]. Shi et al. prepared NIR light-harvesting fullerene-based nanoparticles (DAF NPs) with good biocompatibility for photoacoustic imaging-guided tumor PDT and PTT by nano precipitation method [106]. In addition, Pu et al. used PEG-b-PPG-b-PEG to encapsulate fullerene in 
semiconductor polymer nanoparticles to prepare a molecular diagnostic system for PAI guidance [108]. The findings of these studies showed that photoacoustic and photothermal properties of SP nanoparticles were enhanced after encapsulation with fullerene.

\section{Combination of nuclear magnetic resonance imaging with PDT / PTT for tumor treatment}

Nuclear magnetic resonance imaging (NMRI) is used to detect emitted electromagnetic wave in external gradient magnetic field, obtain the position and type of atomic nucleus in study material, and draw the internal structure image of the object using the energy released from a specific substance to attenuate in different materials. Advantages of MRI including having no harm to the human body and it is a safe, fast and accurate clinical diagnosis method. It provides rich diagnostic information, such as ability to distinguish benign and malignant tumors. Phototherapy induces apoptosis or necrosis of tumor tissues and has low toxicity on normal tissues. MRI is used to detect confirm the location and size of the tumor in vivo and provides diagnostic assistance and real-time monitoring for phototherapy. Several multifunctional composite nanoparticles with photothermal effect and MRI have been developed, which can be used in diagnosis and treatment of tumors.

As a common compound, porphyrin is a pioneer in photodynamic cancer treatment and MRI [109]. The application of porphyrins and their metal derivatives in molecular imaging is a hot topic in recent years. Porphyrin, a first-generation of PS serves as a carrier and has high drug release ability in target cells, therefore it is effectively used in PDT of tumor. Tetranuclear gadolinium (III) porphyrin complexes reported by Luo et al. [110] can be used as therapeutic drugs for MRI and PDT.

In recent years, research on MRI contrast agents has developed rapidly. NMRI contrast agents can be divided into two categories: paramagnetic and superparamagnetic materials [111]. In this review, nano drugs, refer to using nanometer carrier or nanoparticles modified photosensitive material. They make use of superparamagnetic iron oxide nanoparticles (SPIONs) property to improve magnetic resonance imaging and multimode PTT nano drugs in addition to field agent. Furthermore, the agent can provide tumor imaging and are used for combination with chemotherapy drugs [112] (Figure 8). Although now most studies are still in preclinical stages, further clinical environment research on PTT nano drug treatment of CRC should be carried out. Requirements for contrast media are also strict, and include being safe, having no harm the body, and can be improved to reduce toxicity. Combination of specific coordination metal ions with porphyrin, then the ability of RF pulse can be affected, which can be used as MRI contrast agent, the basic standard of MRI contrast agent is to affect RF pulse. Copper, iron, manganese and other metals are inserted into porphyrin respectively. Studies report that manganese complex is the best contrast agent. Therefore, manganese-based contrast agents are prepared by combining manganese with tetraaryl porphyrin cavity [15]. Although some contrast agents have been approved for clinical use, no contrast agents are reported for treatment of malignant tumors.

Multimodal nanomedicines combine different therapies such as NIR light-absorbing nanoparticles to mediate PTT and conventional chemotherapeutic drugs (ie, Doxorubicin, SN38, and Oxaliplatin). Nanomedicines can be functionalized with SPIONs to Facilitate Magnetic Resonance Imaging and Polyethylene Glycol (PEG) to improve biocompatibility and prolong systemic circulation. Cancer-targeting ligands such as antibodies, aptamers, and Hyaluronic Acid (HA) can be conjugated onto nanomedicines to improve delivery and uptake of nanomedicines in cancers [112].

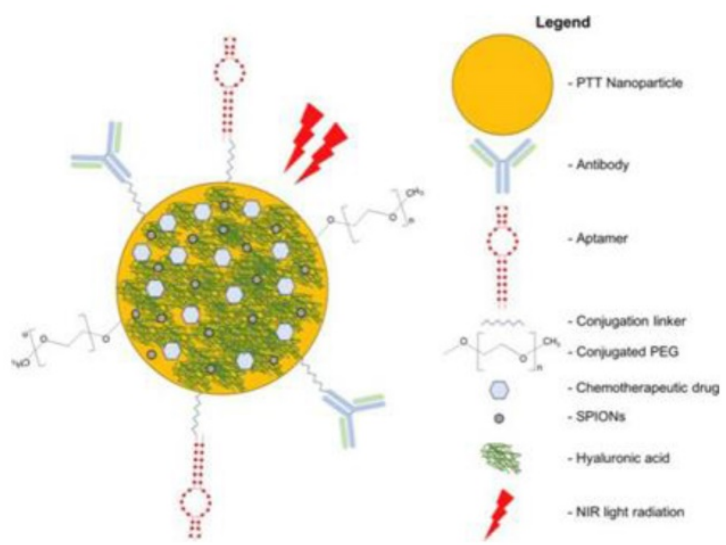

Figure 8. Schematic diagram of multimodal targeted PTT nanomedicine.

\section{Combination of nuclear imaging with PDT I PTT for tumor treatment}

Nuclear imaging technology is a new technology that combines nuclear technology science with modern image theory, including positron emission tomography (PET), single-photon emission tomography (SPECT), X-ray tomography (XCT), nuclear magnetic resonance computed tomography (NMR-CT), and Compton scattering tomography (CST). PET and SPECT with nuclear imaging function are used for tissue imaging and have a deeper tissue 
penetration effect compared with fluorescence imaging. Imaging information is provided to PET and SPECT by detecting radioactive signals of radioisotopes [4]. SPECT uses a $\gamma$ camera to rotate around the human body to sample the whole body or a limited part of the body to achieve tomography imaging. The principle of PET is based on the positron and electron annihilation effect to detect photons formed after annihilation. Cancer cells can be detected with special capabilities of PET, such as radioisotope labeling on PET, and pharmacokinetics of PTA can be monitored by PET or SPECT imaging technology.

Dynamic PET imaging for real-time PDT monitoring is a promising method for study of tumor response. It can detect uptake of PS in tumor sites throughout the treatment period. Real-time dynamic PET is used to distinguish two main mechanisms of PDT: direct cell death and tumor angiogenesis [113]. Dris Kharroubi Lakouas D et al. demonstrated that the absorption of 64Cu-DOTA-biotin-sav after PDT is displayed by PET imaging. SPECT imaging shows absorption of 99mTC-Annexin-V after PDT. Berard et al. used PET imaging based on 2-deoxy-2[(18)F]fluoro-D-glucose(18-FDG) radioactivity to study the effect of dynamic real-time treatment [114, 115]. In addition, different sulfonated phthalocyanine analogs have been used as PSs to evaluate the effect of PDT on tumor 18-FDG uptake. Studies report that direct induction of cell death by 18F-PDG was characterized by a rapid reduction of 18F-PDG uptake, followed by a return to more than $80 \%$ of the initial rate at the end of light exposure. Nuclear medicine is an essential technology in the current tumor phototherapy. Nuclear imaging not only monitors the tumor but also evaluates the uptake rate of PSs in deep tumors to improve treatment.

Although many studies have shown that imaging and PDT / PTT are widely used in tumors, many shortcomings persist, such as single-mode imaging, separate application of PDT or PTT. The outcome is often unsatisfactory due to the defects of each imaging technology and single therapy. Multimodality imaging is the current research direction to integrate the advantages of each imaging mode into one. Zhang et al. [100] studied the PTT-PDT dual therapy system guided by fluorescence $\mathrm{Mr}$ dual-mode imaging, and constructed a new core-shell system, $\mathrm{Fe}_{3} \mathrm{O}_{4} @ \mathrm{C} @$ PMOF Nanocomposites. The results of tumor-bearing mice showed that it had high tumor accumulation and low cytotoxicity.

\section{Synergistic therapy of PTT and PDT}

Although PDT and PTT show great potential in non-invasive and selective treatment of tumors, they have shortcomings when used alone. Synergistic therapy includes combination of chemotherapy and PTT, PDT and chemotherapy synergy, PDT and PTT synergy, and PDT, PTT, and chemotherapy synergy. PDT and PTT combined therapy has a wide potential for application prospect in tumor treatment. Synergy therapy is less toxic and more effective compared with monotherapy. Therefore, several studies are currently exploring synergy therapy, according to the latest literature summary, we get Table 1, which compares and analyzes the characteristics of photodynamic therapy, surgical therapy and chemotherapy and radiotherapy.

\section{Limitations of PTT and PDT}

Most PSs and ablation agents are nanomaterials. However, nanomaterials have certain limitations in phototherapy for tumors including: 1) Most PSs have a hydrophobic structure and have a small solvent degree, making it difficult to generate stable solutions. 2) These compounds are easy to stay and aggregate in the body and have certain physiological toxicity to normal tissues, therefore, targeting needs to be further improved. 3) The infrared absorption efficiency of PSs and generation effect of active oxygen under the action of NIR [116]. 4) Photothermal conversion efficiency of ablative agents should be further improved. 5) Ablative agents have low light stability.

\section{Theoretical basis of combination therapy}

Studies on PDT and PTT report that most of the newly synthesized PSs and ablation agents are nanomaterials, PSs and ablation agents need to function under irradiation of NIR, and mechanisms of cell death induced by the two are similar. Therefore, several studies are currently focusing on the combination therapy of PDT and PTT. Uses of the ablative nanoparticles are used as carrier for PSs alleviates shortcoming of water solubility and light wavelength. Furthermore, PSs can also improve the light-to-heat conversion efficiency of the ablative agent, resulting in an efficient radiation treatment system. Combination of PDT and PTT triggers photothermal effect to enhance the efficiency of PDT, thus producing a synergistic effect of photothermal effect, accelerate blood flow in the tumor, and results in delivery of more oxygen to the tumor tissue, thereby improving efficacy of PDT.

\section{Application of PDT and PTT Combination Therapy}

PSs and ablators are combined in a variety of ways and exhibit good anticancer activity by synergizing photothermal and photodynamic pathways under the action of NIR. Seo SH et al [117]. report that combination of PSs nanoparticles and 
ablator nanoparticles show improved effects. In the study, gold nanorods (GNR) and methylene blue (MB) were used as photothermal materials. Methyl blue is a water-soluble phenothiazine PS with high ${ }^{1} \mathrm{O}_{2}$ yield. GNRs are used as ablators. MB-GNR prolongs NIR irradiation time, produce varieties of ROS compared with a single therapy, exhibit dual effects of PTT and PDT, and have good anticancer activity. Further, in a different study pyrrolopyrrole-triphenyl lamine organic nanoparticles (DPP-TPA) were synthesized for PAI and used in combination with PTT and PDT. In animal experiments, DPP-TPA was injected into subcutaneous tumor-bearing mice. Administration of synergistic therapy, the tumor disappeared completely after treatment.

In a study of Anthony J Trinidad et al [118], gold nanoshells were combined with macrophages to improve delivery for PTT. Macrophages containing gold nanoshells were used to separate human head and neck squamous cell carcinomas. The combination was developed by designing PTT then PS for PDT was added, then the mixture was subjected to $670 \mathrm{~nm}$ and $810 \mathrm{~nm}$ light to explore the synergistic effect of PDT and PTT. Findings of the study showed that the combined treatment reduced the viability of head and neck squamous cells to less than $40 \%$, and PTT and PDT showed significant synergistic effects.

\section{Clinical application of PS in CRC}

Given its low biological toxicity and excellent metabolic dynamics, PS treatment of CRC has a high safety factor, and its more favorable effect is not only can act on the lesion site of local intestinal tissue but also can induce the whole body immune response. Increasing number of studies have been conducted on accelerating the clinical application of PSs. Up to now, many PSs have been used in CRC research. Although still in the experimental stage, its powerful role has attracted wide attention.

In this review, we summarize the clinical research of PSs. (Table 2)

\section{Organic PSs}

DC 473

A novel PS DC 473, especially the CRC cell line
SW 480, was designed and synthesized by Julia Gala de Pablo et al. It is associated with a resonant Tunku Abdul Rahman signal from the Cytochrome C, suggesting that it may be located in or near this cell compartment [9].

HPD

PDT induced by Haematoporphyrin derivative (HpD) showed significant biological effects on the traditional P-glycoprotein after being regulated by verapamil, thereby effectively inhibiting the growth of CRC cell line HRT 18 in vitro [7].

\section{Hypericin}

Laura Mühleisen et al. studied the effect of hypericin on CRC cell line HT-29, and found that hypericin mainly accumulated in the cytoplasm, which may be in the endoplasmic reticulum, Golgi body, lysosome, and the process is dose-dependent and time-dependent. Under the condition of low hypericin concentration and short illumination time, cell antioxidant system can cope with oxidative stress. However, with increasing hypericin amount and irradiation time, the antioxidant system of cells was overloaded by ROS, and the content of oxidized glutathione was no longer sufficient. Hence, hypericin can regulate the transition from apoptosis to necrosis [119].

\section{$\mathrm{PCl}$ of 3-17I-saporin}

Considering the high expression of epithelial cell adhesion molecule (EpCAM) in human CRC cell line, WiDr, Kaja Lund et al. designed a compound PCI of 3-17I-saporin based on photo immuno therapy. A series of experimental results showed that the cytotoxicity induced by photo immuno therapy was stronger than that induced by phototherapy alone, which provided an idea for the combined diagnosis and treatment of CRC [120].

\section{Foslip Idpdt}

By detecting the potential value of foslip ldpdt in establishing a good CRC mouse model, AUR é lie Reinhard et al. found that it can significantly reduce the incidence of cancer associated with colitis by limiting the extravasation of monocytes and neutrophils [121].

Table 1. Comparison of phototherapy and other cancer treatments.

\begin{tabular}{|c|c|c|c|c|c|c|c|}
\hline Tumor treatment & Cytotoxicity & Drug metabolic rate & Selectivity & Security & Traumatic & Synergy & Risk \\
\hline Photodynamic therapy & Low & High & High & High & Low & High & Low \\
\hline Surgical treatment & Secondary & Secondary & Secondary & Low & High & Secondary & High \\
\hline Chemoradiotherapy & High & Low & Low & Low & High & Secondary & High \\
\hline
\end{tabular}


Table 2. Application of PS in PTT / PDT.

\begin{tabular}{|c|c|c|c|}
\hline Number & PS & Product Category & Highest Phase \\
\hline 1 & Padeliporfin potassium & PDT & Launched-2018 \\
\hline 2 & $\begin{array}{l}\text { Aminolevulinic acid hexyl } \\
\text { ester }\end{array}$ & PDT & Launched-2005 \\
\hline 3 & Mono-L-aspartyl chlorin e6 & PDT & Launched-2004 \\
\hline 4 & Temoporfin & PDT & Launched-2002 \\
\hline 5 & Methyl aminolevulinate & PDT & Launched-2001 \\
\hline 6 & $\begin{array}{l}\text { 5-Aminolevulinic acid } \\
\text { hydrochloride }\end{array}$ & PDT & Launched-2000 \\
\hline 7 & Cetuximab-IRDye-700DX & $\begin{array}{l}\text { PDT } \\
\text { Phthalocyanines }\end{array}$ & Pre-Registered \\
\hline 8 & Tin-mesoporphyrin & PDT & Pre-Registered \\
\hline 9 & SNA-001 (Code came) & PTT & Phase III \\
\hline 10 & ACP-SL017 (Code came) & PDT & Phase III \\
\hline 11 & Rose Bengal disodium & PDT & Phase III \\
\hline 12 & Hypericin & PDT & Phase III \\
\hline 13 & inCVAX & $\begin{array}{l}\text { PTT } \\
\text { polymers }\end{array}$ & Phase II/III \\
\hline 14 & $\begin{array}{l}\text { Tin ethyl etiopurpurin } \\
\text { dichloride }\end{array}$ & PDT & Phase II/III \\
\hline 15 & Deuteporfin & $\begin{array}{l}\text { PDT } \\
\text { Porphyrins }\end{array}$ & Phase II \\
\hline 16 & TLD-1433 (Code came) & $\begin{array}{l}\text { PDT } \\
\text { Ruthenium } \\
\text { Complexes }\end{array}$ & Phase II \\
\hline 17 & Fimaporfin/gemcitabine & PDT & Phase II \\
\hline 18 & Exeporfinium chloride & $\begin{array}{l}\text { PDT } \\
\text { Porphyrins }\end{array}$ & Phase II \\
\hline 19 & Photochlor & PDT & Phase II \\
\hline 20 & $\begin{array}{l}\text { Tin (IV) protoporphyrin IX } \\
\text { dichloride }\end{array}$ & $\begin{array}{l}\text { PDT } \\
\text { Porphyrins } \\
\text { Tin Complexes }\end{array}$ & Phase II \\
\hline 21 & Lemuteporphin & $\begin{array}{l}\text { PDT } \\
\text { Porphyrins }\end{array}$ & Phase II \\
\hline 22 & $\begin{array}{l}\text { Lutetium texaphyrin } \\
\text { Motexafin lutetium }\end{array}$ & PDT & Phase II \\
\hline 23 & Redaporfin & PDT & Phase I/II \\
\hline 24 & Fimaporfin/bleomycin & PDT & Phase I/II \\
\hline 25 & Photocyanine & $\begin{array}{l}\text { PDT } \\
\text { Phthalocyanines } \\
\text { Zinc Complexes }\end{array}$ & Phase I \\
\hline 26 & Silicon phthalocyanine 4 & $\begin{array}{l}\text { PDT } \\
\text { Phthalocyanines }\end{array}$ & Phase I \\
\hline 27 & $\begin{array}{l}\text { Aminolevulinic acid benzyl } \\
\text { ester }\end{array}$ & PDT & Phase I \\
\hline 28 & Rhodamine 123 & PDT & Phase I \\
\hline 29 & Fimaporfin & PDT & Clinical \\
\hline 30 & Nanoshells & PTT & Clinical \\
\hline 31 & Sinoporphyrin sodium & $\begin{array}{l}\text { PDT } \\
\text { Porphyrins } \\
\text { Sonodynamic } \\
\text { Therapy (SDT) } \\
\text { Sonosensitizers }\end{array}$ & Preclinical \\
\hline 32 & XF-70 (Code came) & $\begin{array}{l}\text { PDT } \\
\text { Porphyrins }\end{array}$ & Preclinical \\
\hline
\end{tabular}

\section{Talaporfin sodium}

Although the drug-Talaporfin sodium has been approved for marketing in Japan to treat early-stage intrabronchial cancer, the study by Wang et al. is still in the stage of clinical trials. The results of phase I and phase II clinical trials show that the drug is suitable for tumors that are not sensitive to other therapies. In addition, the team's research on the effect of the drug on hepatocellular carcinoma and metastatic CRC is also in phase III clinical trials [122].

\section{Sinoporphyrin sodium}

Bing Zhu et al. observed that sinoporphyrin sodium enhanced the concentration of HCT116 cells and nuclear fragmentation, increased the levels of caspase- 3 and Bax cleavage, inhibited autophagy that promoted cell apoptosis and decreased the size of tumors [123].

\section{Axial disubstituted silicon phthalocyanine}

Ceren SAR synthesized the effect of axial disubstituted silicon phthalocyanine on the PDT of CRC cell line HCT116. Compared with the effect on cancer cells under the condition of no light, the toxic effect on cancer cells caused by different concentrations of photosensitive molecules was examined. It was proved that the drug could induce the corresponding cancer cell toxicity only under the condition of laser irradiation [124].

\section{$\mathrm{F}_{2} \mathrm{BOH}$}

Andre F.S. Luz et al. studied the photodynamic effect of water-soluble Bacillin $\mathrm{F}_{2} \mathrm{BOH}$ on $\mathrm{CT}-26$ mice colon cancer cells and found that the number of apoptotic bodies increased after short-term and low-dose irradiation. After long-term and high-dose irradiation, the scope of cell necrosis was larger. At the same time, the compound can be transported in a non-toxic carrier, thereby reducing the corresponding side effects on organisms [125].

\section{Lu dota peptide 2}

Hossein behnammanesh et al. studied the effect of a new ${ }^{177} \mathrm{Lu}$ labeled somatostatin receptor antagonist, ${ }^{177} \mathrm{Lu}$ dota peptide 2 on human CRC cell line HT-27. As a Somatostatin (SST) radio antagonist, it has high stability in vitro and good affinity to SSTR. Therefore, the drug has broad therapeutic prospects drug for human colorectal adenocarcinoma [126].

\section{Nanomaterial PS NSP-HA-CD}

$\mathrm{Hu}$ Xianli et al. designed a prodrug nano carrier, which integrates hyaluronic acid (HA) targeting CD44 with NLG919 HA and reductive unstable heterodimer into the supramolecular nanocomposite. Under the irradiation of NIR laser, ROS are released, and the antitumor properties of cytotoxic T-lymphocytes are further enhanced. The antitumor effect of cytotoxic T-lymphocytes is obvious in CRC cell line CT-26 [127].

\section{HA - PDA - Ce6}

Xiaoling Wang et al. developed a bimodal HA PDA - Ce6 targeting CRC as a local delivery system and PTT attribute of PDT, which has high photothermal and photodynamic dual activities and 
can accurately reach the tumor site through CD44 mediated endocytosis to achieve the goal of drug accumulation and penetration into deep tissues. HA PDA - Ce6 showed the strongest antitumor effect in HCT-116 mice [128].

\section{$\mathrm{CCPs} / \mathrm{HPPH} / \mathrm{DOX}$}

Weijing Yang et al. reported a dendritic cell vaccine that essentially uses multifunctional polymer vesicles as adjuvants in combination with chemotherapy and PDT. Both DPX and PDT in CCPs / HPPH / DOX molecules can induce ICD. At the same time, the existence of adjuvant can not only enhance the immune recognition effect of the body but also reduce the drug dosage and injection times [129].

\section{Ce6tetraHA}

Shin Jung et al. synthesized a kind of Ce6tetraHA nanocomposite and detected the fluorescence intensity of the drug on mouse CRC cell line HCT116. The drug could be delivered to the tumor site in the way of redox sensitivity and CD44 sensitivity, making the fluorescence intensity of tumor site significantly higher than that of other organs and tissues [130].

\section{Inorganic PS}

\section{8-MOP}

Magdalena bartnik et al. confirmed that 8-MOP could activate endogenous or exogenous apoptosis pathway by inhibiting Akt phosphorylation, thereby significantly suppressing the growth of metastatic colon cancer cell line SW620 [131].

\section{Conclusion}

With the rapid development of bioinformatics technology, the properties and functions of nanoparticles are becoming more and more extensive. The design of nanoparticle as an intelligent tool to carry PSs is one of the research hotspots of precision medicine in the 21st century. Through this operation, researchers have designed a series of biodegradable materials with good biocompatibility and targeting, such as liposomes, organic microspheres and iron oxide. These nanoparticles have achieved remarkable results in a variety of tumor diagnosis and treatment experiments. However, in clinical trials, there are still huge obstacles and challenges in the application of PDT and PTT in deep tissue tumors, for example, there is no light that can reach deep tissue, there is not enough photosensitive material accumulated in deep tissue, or the heat and ROS generated are not enough to achieve the purpose of inhibiting tumor growth. Nanoparticles carrying PS therapy is expected to become a potential treatment for cancer, including colorectal cancer. Many new technologies and strategies have been applied in this field. The surface modified nanoparticles can be well targeted to specific tissues, cells and even organelles. With the help of medical imaging, we can visualize the transport of nanoparticles in the body, and provide a process for PS to play its role. The combination of nanoparticles carrying photosensitive materials with immunotherapy and chemotherapy also contributes to the development of PDT and PTT. In the future, the research of nanoparticles will focus on the safety and effectiveness evaluation of the combination of nanoparticles and PSs. It is also one of the great challenges to combine PDT and PTT with nano medicine to achieve great biological effects by using photosensitive materials loaded with small doses of nanoparticles.

The extensive and multifaceted researchs of PSs show their great application prospect. On the one hand, due to the continuous improvement of PSs own performance and the development of its compatibility with organisms, such as more accurate biological targets and better biological effects, it will be more conducive to the diagnosis and treatment of tumor, and also cater to the medical trend of integration of diagnosis and treatment. On the other hand, with the deepening of interdisciplinary, the relationship between PS and imaging, immunology, nanomaterials and other related disciplines can be further strengthened, and the association between PS and other disciplines such as genetics and other disciplines will also be established and developed in the process of communication.

As mentioned earlier, in order to further optimize the performance of PS, it is feasible to construct nanoparticles with a variety of conjugated polymers. At present, the problem of whether nanoparticles will cause potential damage to the body has not been solved. The next generation of PS research needs to improve the biological characteristics of PS and ensure its biological safety [132]. In addition, we should pay attention to all aspects of the conditions needed for the interaction between PS and organism, such as optimizing the input dose of PS, drug level in tumor, light source and tissue oxygen conditions [133]. What is more needed is to change to clinical related research field or even clinical under the condition of ensuring safety [134]. Perhaps, in the future, researchers can establish a large database about photothermal based nanoparticles to realize the rapid change of PS industry. 


\section{Abbreviations}

PTT: Photothermal therapy; PDT: Photodynamic therapy; NIR: Near-infrared; PSs: Photosensitizers; PS: Photosensitizer; ${ }^{1} \mathrm{O}_{2}$ : Singlet oxygen; HPD: Hematophorphyrin derivative; ROS: Reactive oxygen species; MB: Methylene blue; DPP-TPA: Pyrrolopyrrole-triphenyl lamine organic nanoparticles; PAI: Photoacoustic imaging; PAT: Photoacoustic tomography; GN: Gold nanorods; WP5: Column aromatic hydrocarbon; 5-ALA: 5-aminolevulinic acid hydrochloride; NMRI: Nuclear magnetic resonance imaging; SPIONs: Superparamagnetic iron oxide nanoparticles; PET: Positron emission tomography; SPECT: Single-photon emission tomography; SPR: Surface plasmon resonance; 18-FDG: 2-deoxy-2[(18)F]fluoro-D-glucose; GNR: Gold nanorods; PDA NPs: Polydopamine nanoparticles.

\section{Competing Interests}

The authors have declared that no competing interest exists.

\section{Acknowledgments}

This work is supported by the National Natural Science Foundation of China (No. 81803573), China Postdoctoral Science Foundation (No. 2018M640672), Key R\&D and Promotion Projects in Henan Province (Nos. 202102310155 and 212102310874), the Key Scientific Research Program for Universities of Henan Province (No. 18B180005), College Students' Innovative Entrepreneurial Training Plan Program (No. 202010475090).

\section{References}

1. Bray F, Ferlay J, Soerjomataram I, Siegel RL, Torre LA, Jemal A. Global cancer statistics 2018: GLOBOCAN estimates of incidence and mortality worldwide for 36 cancers in 185 countries. CA: a cancer journal for clinicians. 2018; 68: 394-424.

2. Hu D, Chen L, Qu Y, Peng J, Chu B, Shi K, et al. Oxygen-generating Hybrid Polymeric Nanoparticles with Encapsulated Doxorubicin and Chlorin e6 for Trimodal Imaging-Guided Combined Chemo-Photodynamic Therapy. Theranostics. 2018; 8: 1558-74.

3. Zhang L, Liu W, Liu F, Wang Q, Song M, Yu Q, et al. IMCA Induces Ferroptosis Mediated by SLC7A11 through the AMPK/mTOR Pathway in Colorectal Cancer. Oxidative medicine and cellular longevity. 2020; 2020: 1675613.

4. Shi X, Zhang $\mathrm{H}$, Jin $\mathrm{W}$, Liu $\mathrm{W}$, Yin $\mathrm{H}, \mathrm{Li} \mathrm{Y}$, et al. Metronomic photodynamic therapy with 5 -aminolevulinic acid induces apoptosis and autophagy in human SW837 colorectal cancer cells. J Photochem Photobiol B. 2019; 198: 111586.

5. Li Y, Xin J, Sun Y, Han T, Zhang H, An F. Magnetic resonance imaging-guided and targeted theranostics of colorectal cancer. Cancer biology \& medicine. 2020; 17: 307-27.

6. Abdelghany SM, Schmid D, Deacon J, Jaworski J, Fay F, McLaughlin $\mathrm{KM}$, et al. Enhanced antitumor activity of the photosensitizer meso-Tetra(N-methyl-4-pyridyl) porphine tetra tosylate through encapsulation in antibody-targeted chitosan/alginate nanoparticles. Biomacromolecules. 2013; 14: 302-10.

7. Purkiss SF, Grahn MF, Williams NS. Haematoporphyrin derivative--photodynamic therapy of colorectal carcinoma, sensitized using verapamil and adriamycin. Surgical oncology. 1996; 5: 169-75.

8. Kawczyk-Krupka A, Bugaj AM, Latos W, Zaremba K, Wawrzyniec K, Kucharzewski M, et al. Photodynamic therapy in colorectal cancer
treatment--The state of the art in preclinical research. Photodiagnosis Photodyn Ther. 2016; 13: 158-74.

9. Gala de Pablo J, Chisholm DR, Steffen A, Nelson AK, Mahler C, Marder $\mathrm{TB}$, et al. Tandem fluorescence and Raman (fluoRaman) characterisation of a novel photosensitiser in colorectal cancer cell line SW480. The Analyst. 2018; 143: 6113-20.

10. Mu C, Wang W, Wang J, Gong C, Zhang D, Zhang X. Probe-Free Direct Identification of Type I and Type II Photosensitized Oxidation Using Field-Induced Droplet Ionization Mass Spectrometry. Angew Chem Int Ed Engl. 2020; 59: 21515-9.

11. Estébanez S, Lorente C, Kaufman TS, Larghi EL, Thomas AH, Serrano MP. Photophysical and Photochemical Properties of 3-methylpterin as a New and More Stable Pterin-type Photosensitizer. Photochemistry and photobiology. 2018; 94: 881-9.

12. Baptista MS, Cadet J, Di Mascio P, Ghogare AA, Greer A, Hamblin MR, et al. Type I and Type II Photosensitized Oxidation Reactions: Guidelines and Mechanistic Pathways. Photochemistry and photobiology. 2017; 93: 912-9.

13. Nath S, Saad MA, Pigula M, Swain JWR, Hasan T. Photoimmunotherapy of Ovarian Cancer: A Unique Niche in the Management of Advanced Disease. Cancers. 2019; 11.

14. Chen J, Zeng Z, Huang L, Luo S, Dong J, Zhou FH, et al. Photothermal therapy technology of metastatic colorectal cancer. American journal of translational research. 2020; 12: 3089-115.

15. Kwiatkowski S, Knap B, Przystupski D, Saczko J, Kędzierska E, Knap-Czop K, et al. Photodynamic therapy - mechanisms, photosensitizers and combinations. Biomedicine \& pharmacotherapy $=$ Biomedecine \& pharmacotherapie. 2018; 106: 1098-107.

16. Allison RR, Sibata CH. Oncologic photodynamic therapy photosensitizers: a clinical review. Photodiagnosis and photodynamic therapy. 2010; 7: 61-75.

17. Yoon I, Li JZ, Shim YK. Advance in photosensitizers and light delivery for photodynamic therapy. Clinical endoscopy. 2013; 46: 7-23.

18. Hirohara S, Oka C, Totani M, Obata M, Yuasa J, Ito H, et al. Synthesis, Photophysical Properties, and Biological Evaluation of trans-Bisthioglycosylated Tetrakis(fluorophenyl)chlorin for Photodynamic Therapy. Journal of medicinal chemistry. 2015; 58: 8658-70.

19. Li X, Liu L, Li S, Wan Y, Chen JX, Tian S, et al. Biodegradable $\Pi$-Conjugated Oligomer Nanoparticles with High Photothermal Conversion Efficiency for Cancer Theranostics. ACS Nano. 2019; 13: 12901-11.

20. Li X, Park EY, Kang Y, Kwon N, Yang M, Lee S, et al. Supramolecular Phthalocyanine Assemblies for Improved Photoacoustic Imaging and Photothermal Therapy. 2020; 59: 8630-4.

21. Huang X, El-Sayed IH, Qian W, El-Sayed MA. Cancer cell imaging and photothermal therapy in the near-infrared region by using gold nanorods. Journal of the American Chemical Society. 2006; 128: 2115-20.

22. Skrabalak SE, Chen J, Sun Y, Lu X, Au L, Cobley CM, et al. Gold nanocages: synthesis, properties, and applications. Accounts of chemical research. 2008; 41: 1587-95.

23. Chen J, Glaus C, Laforest R, Zhang Q, Yang M, Gidding M, et al. Gold nanocages as photothermal transducers for cancer treatment. Small (Weinheim an der Bergstrasse, Germany). 2010; 6: 811-7.

24. Kim JW, Galanzha EI, Shashkov EV, Moon HM, Zharov VP. Golden carbon nanotubes as multimodal photoacoustic and photothermal high-contrast molecular agents. Nature nanotechnology. 2009; 4: 688-94.

25. Xie X, Li Z, Zhang Y, Guo S, Pendharkar AI, Lu M, et al. Emerging $\approx 800$ nm Excited Lanthanide-Doped Upconversion Nanoparticles. Small (Weinheim an der Bergstrasse, Germany). 2017; 13.

26. Zhang $\mathrm{P}, \mathrm{Hu} \mathrm{C}$, Ran $\mathrm{W}$, Meng J, Yin $\mathrm{Q}, \mathrm{Li}$ Y. Recent Progress in Light-Triggered Nanotheranostics for Cancer Treatment. Theranostics. 2016; 6: 948-68.

27. Chu WY, Tsai MH, Peng CL, Shih YH, Luo TY, Yang SJ, et al. pH-Responsive Nanophotosensitizer for an Enhanced Photodynamic Therapy of Colorectal Cancer Overexpressing EGFR. Mol Pharm. 2018; 15: $1432-44$.

28. Yang L, He J, Wen Y, Yi W, Li Q, Lin L, et al. Nanoscale Photodynamic Agents for Colorectal Cancer Treatment: A Review. Journal of biomedical nanotechnology. 2016; 12: 1348-73.

29. Tang X, Tan L, Shi K, Peng J, Xiao Y, Li W, et al. Gold nanorods together with HSP inhibitor-VER-155008 micelles for colon cancer mild-temperature photothermal therapy. Acta Pharm Sin B. 2018; 8: 587-601.

30. Nicolodelli G, Kurachi C, Rego RF, Omairi T, Bagnato VS. Evidence of 5 -aminolevulinic acid (ALA) penetration increase due to microdrilling in soft tissue using femtosecond laser ablation. Lasers Med Sci. 2012; 27: 1067-71. 
31. Ai X, Mu J, Xing B. Recent Advances of Light-Mediated Theranostics. Theranostics. 2016; 6: 2439-57.

32. Bansal A, Zhang Y. Photocontrolled nanoparticle delivery systems for biomedical applications. Accounts of chemical research. 2014; 47: 3052-60.

33. Warburg O. On the origin of cancer cells. Science (New York, NY). 1956; 123: 309-14.

34. Lotan R, Raz A. Lectins in cancer cells. Annals of the New York Academy of Sciences. 1988; 551: 385-96; discussion 96-8.

35. Liu Z, Gao Y, Jin X, Deng Q, Yin Z, Tong $S$, et al. Regioisomer-manipulating thio-perylenediimide nanoagents for photothermal/photodynamic theranostics. J Mater Chem B. 2020; 8: 5535-44.

36. Chatterjee DK, Fong LS, Zhang Y. Nanoparticles in photodynamic therapy: an emerging paradigm. Advanced drug delivery reviews. 2008; 60: $1627-37$

37. Doane TL, Burda C. The unique role of nanoparticles in nanomedicine: imaging, drug delivery and therapy. Chemical Society reviews. 2012; 41: 2885-911.

38. Dykman L, Khlebtsov N. Gold nanoparticles in biomedical applications: recent advances and perspectives. Chemical Society reviews. 2012; 41: 2256-82.

39. Khot MI, Andrew H, Svavarsdottir HS, Armstrong G, Quyn AJ, Jayne DG. A Review on the Scope of Photothermal Therapy-Based Nanomedicines in Preclinical Models of Colorectal Cancer. Clin Colorectal Cancer. 2019; 18: e200-e9.

40. Shao L, Pan Y, Hua B, Xu S, Yu G, Wang M, et al. Constructing Adaptive Photosensitizers via Supramolecular Modification Based on Pillararene Host-Guest Interactions. Angew Chem Int Ed Engl. 2020; 59: 11779-83.

41. Wang Y, Luo S, Wu Y, Tang P, Liu J, Liu Z, et al. Highly Penetrable and On-Demand Oxygen Release with Tumor Activity Composite Nanosystem for Photothermal/Photodynamic Synergetic Therapy. ACS Nano. 2020.

42. Ou K, Kang Y, Chen L, Zhang X, Chen X, Zheng Y, et al. $\mathrm{H}(2) \mathrm{O}(2)$-responsive nano-prodrug for podophyllotoxin delivery. Biomaterials science. 2019; 7: 2491-8.

43. Gao J, Li J, Geng WC, Chen FY, Duan X, Zheng Z, et al. Biomarker Displacement Activation: A General Host-Guest Strategy for Targeted Phototheranostics in Vivo. Journal of the American Chemical Society. 2018; 140: 4945-53.

44. Wang $\mathrm{C}$, Zhao N, Yuan W. NIR/Thermoresponsive Injectable Self-Healing Hydrogels Containing Polydopamine Nanoparticles for Efficient Synergistic Cancer Thermochemotherapy. ACS applied materials \& interfaces. 2020; 12: 9118-31.

45. Zhang Y, Wang B, Zhao R, Zhang Q, Kong X. Multifunctional nanoparticles as photosensitizer delivery carriers for enhanced photodynamic cancer therapy. Materials science \& engineering C, Materials for biological applications. 2020; 115: 111099.

46. Abrantes ABP, Dias GC, Souza-Pinto NC, Baptista MS. p53-Dependent and p53-Independent Responses of Cells Challenged by Photosensitization. Photochemistry and photobiology. 2019; 95: 355-63.

47. Kim JK, Byun MR, Maeng CH, Kim YR, Choi JW. Selective Targeting of Cancer Stem Cells (CSCs) Based on Photodynamic Therapy (PDT) Penetration Depth Inhibits Colon Polyp Formation in Mice. Cancers. 2020; 12

48. Andraos C, Gulumian M. Intracellular and extracellular targets as mechanisms of cancer therapy by nanomaterials in relation to their physicochemical properties. Wiley interdisciplinary reviews Nanomedicine and nanobiotechnology. 2020: e1680.

49. Ni K, Lan G, Veroneau SS, Duan X, Song Y, Lin W. Nanoscale metal-organic frameworks for mitochondria-targeted radiotherapy-radiodynamic therapy. Nat Commun. 2018; 9: 4321.

50. Akter S, Inai M, Saito S, Honda N, Hazama H, Nishikawa T, et al. Photodynamic therapy by lysosomal-targeted drug delivery using talaporfin sodium incorporated into inactivated virus particles. Laser therapy. 2019; 28: 245-56.

51. Nam JS, Kang MG, Kang J, Park SY, Lee SJ, Kim HT, et al. Endoplasmic Reticulum-Localized Iridium(III) Complexes as Efficient Photodynamic Therapy Agents via Protein Modifications. Journal of the American Chemical Society. 2016; 138: 10968-77.

52. Pang $W$, Jiang $P$, Ding $S$, Bao $Z$, Wang $N$, Wang $H$, et al. Nucleolus-Targeted Photodynamic Anticancer Therapy Using Renal-Clearable Carbon Dots. Advanced healthcare materials. 2020; 9: e2000607.

53. Sarasqueta AF, Forte G, Corver WE, de Miranda NF, Ruano D, van Eijk $\mathrm{R}$, et al. Integral analysis of p53 and its value as prognostic factor in sporadic colon cancer. BMC cancer. 2013; 13: 277.

54. Sanz-Garcia E, Argiles G, Elez E, Tabernero J. BRAF mutant colorectal cancer: prognosis, treatment, and new perspectives. Annals of oncology : official journal of the European Society for Medical Oncology. 2017; 28: 2648-57.

55. Karpisheh V, Nikkhoo A, Hojjat-Farsangi M, Namdar A, Azizi G, Ghalamfarsa G, et al. Prostaglandin E2 as a potent therapeutic target for treatment of colon cancer. Prostaglandins \& other lipid mediators. 2019; 144: 106338.

56. Majdalawieh AF, Massri M, Ro HS. AEBP1 is a Novel Oncogene: Mechanisms of Action and Signaling Pathways. Journal of oncology. 2020; 2020: 8097872.

57. Ramesh P, Medema JP. BCL-2 family deregulation in colorectal cancer: potential for $\mathrm{BH} 3$ mimetics in therapy. Apoptosis : an international journal on programmed cell death. 2020; 25: 305-20.

58. Kooshkaki O, Derakhshani A, Safarpour H, Najafi S, Vahedi P, Brunetti $\mathrm{O}$, et al. The Latest Findings of PD-1/PD-L1 Inhibitor Application in Gynecologic Cancers. International journal of molecular sciences. 2020;

59. Martinelli E, Ciardiello D, Martini G, Troiani T, Cardone C, Vitiello PP, et al. Implementing anti-epidermal growth factor receptor (EGFR) therapy in metastatic colorectal cancer: challenges and future perspectives. Annals of oncology : official journal of the European Society for Medical Oncology. 2020; 31: 30-40.

60. Chan DLH, Segelov E, Wong RS, Smith A, Herbertson RA, Li BT, et al. Epidermal growth factor receptor (EGFR) inhibitors for metastatic colorectal cancer. The Cochrane database of systematic reviews. 2017; 6: Cd007047.

61. Wang $\mathrm{LV}, \mathrm{Hu} \mathrm{S}$. Photoacoustic tomography: in vivo imaging from organelles to organs. Science (New York, NY). 2012; 335: 1458-62.

62. Milgrom LR. Towards recombinant antibody-fragment targeted photodynamic therapy. Science progress. 2008; 91: 241-63.

63. Plaetzer K, Krammer B, Berlanda J, Berr F, Kiesslich T. Photophysics and photochemistry of photodynamic therapy: fundamental aspects. Lasers in medical science. 2009; 24: 259-68.

64. Dysart JS, Patterson MS. Characterization of Photofrin photobleaching for singlet oxygen dose estimation during photodynamic therapy of MLL cells in vitro. Physics in medicine and biology. 2005; 50: 2597-616

65. Kochevar IE. Singlet oxygen signaling: from intimate to global. Science's STKE : signal transduction knowledge environment. 2004; 2004: pe7.

66. Wagner M, Suarez ER, Theodoro TR, Machado Filho CD, Gama MF, Tardivo JP, et al. Methylene blue photodynamic therapy in malignant melanoma decreases expression of proliferating cell nuclear antigen and heparanases. Clinical and experimental dermatology. 2012; 37: 527-33.

67. Chiu SM, Oleinick NL. Dissociation of mitochondrial depolarization from cytochrome $\mathrm{c}$ release during apoptosis induced by photodynamic therapy. British journal of cancer. 2001; 84: 1099-106.

68. Barge J, Decréau R, Julliard M, Hubaud JC, Sabatier AS, Grob JJ, et al. Killing efficacy of a new silicon phthalocyanine in human melanoma cells treated with photodynamic therapy by early activation of mitochondrion-mediated apoptosis. Experimental dermatology. 2004; 13: 33-44.

69. Zou Z, Chang H, Li H, Wang S. Induction of reactive oxygen species: an emerging approach for cancer therapy. Apoptosis : an international journal on programmed cell death. 2017; 22: 1321-35.

70. Chiu SM, Xue LY, Lam M, Rodriguez ME, Zhang P, Kenney ME, et al. A requirement for bid for induction of apoptosis by photodynamic therapy with a lysosome- but not a mitochondrion-targeted photosensitizer. Photochemistry and photobiology. 2010; 86: 1161-73.

71. Tian J, Ding L, Xu HJ, Shen Z, Ju H, Jia L, et al. Cell-specific and $\mathrm{pH}$-activatable rubyrin-loaded nanoparticles for highly selective near-infrared photodynamic therapy against cancer. Journal of the American Chemical Society. 2013; 135: 18850-8.

72. Igney FH, Krammer PH. Death and anti-death: tumour resistance to apoptosis. Nature reviews Cancer. 2002; 2: 277-88.

73. Mori M, Kuroda T, Obana A, Sakata I, Hirano T, Nakajima S, et al. In vitro plasma protein binding and cellular uptake of ATX-S10(Na), a hydrophilic chlorin photosensitizer. Japanese journal of cancer research : Gann. 2000; 91: 845-52.

74. Gomer CJ. Preclinical examination of first and second generation photosensitizers used in photodynamic therapy. Photochemistry and photobiology. 1991; 54: 1093-107.

75. Skinner SA, Tutton PJ, O'Brien PE. Microvascular architecture of experimental colon tumors in the rat. Cancer research. 1990; 50: 2411-7.

76. Henderson BW, Waldow SM, Mang TS, Potter WR, Malone PB, Dougherty TJ. Tumor destruction and kinetics of tumor cell death in two experimental mouse tumors following photodynamic therapy. Cancer research. 1985; 45: 572-6.

77. Korbelik M, Dougherty GJ. Photodynamic therapy-mediated immune response against subcutaneous mouse tumors. Cancer research. 1999; 59: 1941-6. 
78. Gollnick SO, Vaughan L, Henderson BW. Generation of effective antitumor vaccines using photodynamic therapy. Cancer research. 2002; 62: 1604-8.

79. Nowak RM, Jacobsen G, Christenson RH, Moyer M, Hudson M, McCord J. Differentiating type 1 and 2 acute myocardial infarctions using the $\mathrm{N}$-terminal pro B-type natriuretic peptide/cardiac troponin $\mathrm{T}$ ratio. The American journal of emergency medicine. 2018; 36: 1849-54.

80. Frei A, Rubbiani R, Tubafard S, Blacque O, Anstaett $\mathrm{P}$, Felgenträger A, et al. Synthesis, characterization, and biological evaluation of new $\mathrm{Ru}$ (II) polypyridyl photosensitizers for photodynamic therapy. Journal of medicinal chemistry. 2014; 57: 7280-92.

81. Chen J, Ning C, Zhou Z, Yu P, Zhu Y, Tan G, et al. Nanomaterials as photothermal therapeutic agents. Progress in materials science. 2019; 99: $1-26$.

82. Hunt CR, Pandita RK, Laszlo A, Higashikubo R, Agarwal M, Kitamura $\mathrm{T}$, et al. Hyperthermia activates a subset of ataxia-telangiectasia mutated effectors independent of DNA strand breaks and heat shock protein 70 status. Cancer research. 2007; 67: 3010-7.

83. Sawaji Y, Sato T, Takeuchi A, Hirata M, Ito A. Anti-angiogenic action of hyperthermia by suppressing gene expression and production of tumour-derived vascular endothelial growth factor in vivo and in vitro. British journal of cancer. 2002; 86: 1597-603.

84. Li S, Deng Q, Zhang Y, Li X, Wen G, Cui X, et al. Rational Design of Conjugated Small Molecules for Superior Photothermal Theranostics in the NIR-II Biowindow. Advanced materials (Deerfield Beach, Fla). 2020; 32: e2001146.

85. Zharov VP, Galitovskaya EN, Johnson C, Kelly T. Synergistic enhancement of selective nanophotothermolysis with gold nanoclusters: potential for cancer therapy. Lasers in surgery and medicine. 2005; 37: 219-26.

86. Hosseinzadeh H, Atyabi F, Varnamkhasti BS, Hosseinzadeh R, Ostad $\mathrm{SN}$, Ghahremani $\mathrm{MH}$, et al. SN38 conjugated hyaluronic acid gold nanoparticles as a novel system against metastatic colon cancer cells. International journal of pharmaceutics. 2017; 526: 339-52.

87. Kim J, Park J, Kim H, Singha K, Kim WJ. Transfection and intracellular trafficking properties of carbon dot-gold nanoparticle molecular assembly conjugated with PEI-pDNA. Biomaterials. 2013; 34: 7168-80.

88. Hu M, Chen J, Li ZY, Au L, Hartland GV, Li X, et al. Gold nanostructures: engineering their plasmonic properties for biomedical applications. Chemical Society reviews. 2006; 35: 1084-94.

89. Wang D, Xu Z, Yu H, Chen X, Feng B, Cui Z, et al. Treatment of metastatic breast cancer by combination of chemotherapy and photothermal ablation using doxorubicin-loaded DNA wrapped gold nanorods. Biomaterials. 2014; 35: 8374-84.

90. Fiorica C, Mauro N. Double-Network-Structured Graphene Oxide-Containing Nanogels as Photothermal Agents for the Treatment of Colorectal Cancer. 2017; 18: 1010-8.

91. Arsawang U, Saengsawang O, Rungrotmongkol T, Sornmee $\mathrm{P}$, Wittayanarakul K, Remsungnen $\mathrm{T}$, et al. How do carbon nanotubes serve as carriers for gemcitabine transport in a drug delivery system? Journal of molecular graphics \& modelling. 2011; 29: 591-6.

92. Markovic ZM, Harhaji-Trajkovic LM, Todorovic-Markovic BM, Kepić $\mathrm{DP}$, Arsikin KM, Jovanović SP, et al. In vitro comparison of the photothermal anticancer activity of graphene nanoparticles and carbon nanotubes. Biomaterials. 2011; 32: 1121-9.

93. Liu X, Tao H, Yang K, Zhang S, Lee ST, Liu Z. Optimization of surface chemistry on single-walled carbon nanotubes for in vivo photothermal ablation of tumors. Biomaterials. 2011; 32: 144-51.

94. Koo JS, Lee SY, Nam S, Azad MOK, Kim M, Kim K, et al. Preparation of cupric sulfate-based self-emulsifiable nanocomposites and their application to the photothermal therapy of colon adenocarcinoma. Biochemical and biophysical research communications. 2018; 503: 2471-7.

95. Li KC, Chu HC, Lin Y, Tuan HY, Hu YC. PEGylated Copper Nanowires as a Novel Photothermal Therapy Agent. ACS applied materials \& interfaces. 2016; 8: 12082-90.

96. Hessel CM, Pattani VP, Rasch M, Panthani MG, Koo B, Tunnell JW, et al. Copper selenide nanocrystals for photothermal therapy. Nano letters. 2011; 11: 2560-6.

97. Jung HS, Verwilst P, Sharma A, Shin J, Sessler JL, Kim JS. Organic molecule-based photothermal agents: an expanding photothermal therapy universe. Chemical Society reviews. 2018; 47: 2280-97.

98. Lee SM, Kim HJ, Kim SY, Kwon MK, Kim S, Cho A, et al. Drug-loaded gold plasmonic nanoparticles for treatment of multidrug resistance in cancer. Biomaterials. 2014; 35: 2272-82.

99. Xiao YF, Xiang C, Li S, Mao C, Chen H, Chen JX, et al. Single-Photomolecular Nanotheranostics for Synergetic Near-Infrared Fluorescence and Photoacoustic Imaging-Guided Highly Effective Photothermal Ablation. Small (Weinheim an der Bergstrasse, Germany). 2020; 16: e2002672.
100. Zhang H, Li YH, Chen Y, Wang MM, Wang XS, Yin XB. Fluorescence and Magnetic Resonance Dual-Modality Imaging-Guided Photothermal and Photodynamic Dual-Therapy with Magnetic Porphyrin-Metal Organic Framework Nanocomposites. Scientific reports. 2017; 7: 44153.

101. Lin J, Wang S, Huang P, Wang Z, Chen S, Niu G, et al. Photosensitizer-loaded gold vesicles with strong plasmonic coupling effect for imaging-guided photothermal/photodynamic therapy. ACS nano. 2013; 7: 5320-9.

102. Pansare V, Hejazi S, Faenza W, Prud'homme RK. Review of Long-Wavelength Optical and NIR Imaging Materials: Contrast Agents, Fluorophores and Multifunctional Nano Carriers. Chemistry of materials : a publication of the American Chemical Society. 2012; 24: 812-27.

103. Taratula O, Schumann C, Duong T, Taylor KL, Taratula O. Dendrimer-encapsulated naphthalocyanine as a single agent-based theranostic nanoplatform for near-infrared fluorescence imaging and combinatorial anticancer phototherapy. Nanoscale. 2015; 7: 3888-902.

104. Singh AK, Hahn MA, Gutwein LG, Rule MC, Knapik JA, Moudgil BM, et al. Multi-dye theranostic nanoparticle platform for bioimaging and cancer therapy. International journal of nanomedicine. 2012; 7: 2739-50.

105. Liu Y, Bhattarai P, Dai Z, Chen X. Photothermal therapy and photoacoustic imaging via nanotheranostics in fighting cancer. Chemical Society reviews. 2019; 48: 2053-108.

106. Shi H, Gu R, Xu W, Huang H, Xue L, Wang W, et al. Near-Infrared Light-Harvesting Fullerene-Based Nanoparticles for Promoted Synergetic Tumor Phototheranostics. 2019; 11: 44970-7.

107. Weissleder R, Pittet MJ. Imaging in the era of molecular oncology. Nature. 2008; 452: 580-9.

108. Lyu Y, Fang Y, Miao Q, Zhen X, Ding D, Pu K. Intraparticle Molecular Orbital Engineering of Semiconducting Polymer Nanoparticles as Amplified Theranostics for in Vivo Photoacoustic Imaging and Photothermal Therapy. ACS nano. 2016; 10: 4472-81.

109. Imran M, Ramzan M, Qureshi AK, Khan MA, Tariq M. Emerging Applications of Porphyrins and Metalloporphyrins in Biomedicine and Diagnostic Magnetic Resonance Imaging. Biosensors. 2018; 8 .

110. Luo J, Chen LF, Hu P, Chen ZN. Tetranuclear gadolinium(III) porphyrin complex as a theranostic agent for multimodal imaging and photodynamic therapy. Inorganic chemistry. 2014; 53: 4184-91.

111. Melton DL, Vanderveer DG, Hancock RD. Complexes of greatly enhanced thermodynamic stability and metal ion size-based selectivity, formed by the highly preorganized non-macrocyclic ligand 1,10-phenanthroline-2,9-

dicarboxylic acid. A thermodynamic and crystallographic study. Inorganic chemistry. 2006; 45: 9306-14.

112. Yang SJ, Lin FH, Tsai KC, Wei MF, Tsai HM, Wong JM, et al. Folic acid-conjugated chitosan nanoparticles enhanced protoporphyrin IX accumulation in colorectal cancer cells. Bioconjugate chemistry. 2010; 21: 679-89.

113. Kharroubi Lakouas D, Huglo D, Mordon S, Vermandel M. Nuclear medicine for photodynamic therapy in cancer: Planning, monitoring and nuclear PDT. Photodiagnosis and photodynamic therapy. 2017; 18: 236-43.

114. Byrne AT, O'Connor AE, Hall M, Murtagh J, O'Neill K, Curran KM, et al. Vascular-targeted photodynamic therapy with BF2-chelated Tetraaryl-Azadipyrromethene agents: a multi-modality molecular imaging approach to therapeutic assessment. British journal of cancer. 2009; 101: 1565-73.

115. Bérard V, Rousseau JA, Cadorette J, Hubert L, Bentourkia M, van Lier JE, et al. Dynamic imaging of transient metabolic processes by small-animal PET for the evaluation of photosensitizers in photodynamic therapy of cancer. Journal of nuclear medicine : official publication, Society of Nuclear Medicine. 2006; 47: 1119-26.

116. Zhu H, Cheng P, Chen P, Pu K. Recent progress in the development of near-infrared organic photothermal and photodynamic nanotherapeutics. Biomaterials science. 2018; 6: 746-65.

117. Seo SH, Kim BM, Joe A, Han HW, Chen X, Cheng Z, et al. NIR-light-induced surface-enhanced Raman scattering for detection and photothermal/photodynamic therapy of cancer cells using methylene blue-embedded gold nanorod@SiO2 nanocomposites. Biomaterials. 2014; 35: 3309-18.

118. Trinidad AJ, Hong SJ, Peng Q, Madsen SJ, Hirschberg H. Combined concurrent photodynamic and gold nanoshell loaded macrophage-mediated photothermal therapies: an in vitro study on squamous cell head and neck carcinoma. Lasers in surgery and medicine. 2014; 46: 310-8.

119. Mühleisen L, Alev M, Unterweger H, Subatzus D, Pöttler M, Friedrich $\mathrm{RP}$, et al. Analysis of Hypericin-Mediated Effects and Implications for Targeted Photodynamic Therapy. International journal of molecular sciences. 2017; 18 . 
120. Lund K, Bostad M, Skarpen E, Braunagel M, Kiprijanov S, Krauss S, et al. The novel EpCAM-targeting monoclonal antibody 3-17I linked to saporin is highly cytotoxic after photochemical internalization in breast, pancreas and colon cancer cell lines. mAbs. 2014; 6: 1038-50.

121. Reinhard A, Bressenot A, Dassonneville R, Loywick A, Hot D, Audebert $\mathrm{C}$, et al. Photodynamic therapy relieves colitis and prevents colitis-associated carcinogenesis in mice. Inflammatory bowel diseases. 2015; 21: 985-95.

122. Wang S, Bromley E, Xu L, Chen JC, Keltner L. Talaporfin sodium. Expert opinion on pharmacotherapy. 2010; 11: 133-40.

123. Zhu B, Li S, Yu L, Hu W, Sheng D, Hou J, et al. Inhibition of Autophagy with Chloroquine Enhanced Sinoporphyrin Sodium Mediated Photodynamic Therapy-induced Apoptosis in Human Colorectal Cancer Cells. International journal of biological sciences. 2019; 15: 12-23.

124. Sarı C, Eyüpoğlu FC, Değirmencioğlu İ, Bayrak R. Synthesis of axially disubstituted silicon phthalocyanines and investigation of photodynamic effects on HCT-116 colorectal cancer cell line. Photodiagnosis and photodynamic therapy. 2018; 23: 83-8.

125. Luz AFS, Pucelik B, Pereira MM, Dąbrowski JM, Arnaut LG. Translating phototherapeutic indices from in vitro to in vivo photodynamic therapy with bacteriochlorins. Lasers in surgery and medicine. 2018; 50: 451-9.

126. Behnammanesh H, Erfani M, Hajiramezanali M, Jokar S, Geramifar P, Sabzevari O, et al. Preclinical study of a new (177)Lu-labeled somatostatin receptor antagonist in HT-29 human colorectal cancer cells. Asia Oceania journal of nuclear medicine \& biology. 2020; 8: 109-15.

127. Hu X, Hou B, Xu Z, Saeed M, Sun F, Gao Z, et al. Supramolecular Prodrug Nanovectors for Active Tumor Targeting and Combination Immunotherapy of Colorectal Cancer. Advanced science (Weinheim, Baden-Wurttemberg, Germany). 2020; 7: 1903332.

128. Wang X, Ouyang X, Chen J, Hu Y, Sun X, Yu Z. Nanoparticulate photosensitizer decorated with hyaluronic acid for photodynamic/photothermal cancer targeting therapy. Nanomedicine (London, England). 2019; 14: 151-67.

129. Yang W, Zhu G, Wang S, Yu G, Yang Z, Lin L, et al. In Situ Dendritic Cell Vaccine for Effective Cancer Immunotherapy. ACS Nano. 2019; 13: 3083-94.

130. Jung S, Jung S, Kim DM, Lim SH, Shim YH, Kwon H, et al. Hyaluronic Acid-Conjugated with Hyperbranched Chlorin e6 Using Disulfide Linkage and Its Nanophotosensitizer for Enhanced Photodynamic Therapy of Cancer Cells. Materials (Basel, Switzerland). 2019; 12.

131. Bartnik M, Sławińska-Brych A, Żurek A, Kandefer-Szerszeń M, Zdzisińska B. 8-methoxypsoralen reduces AKT phosphorylation, induces intrinsic and extrinsic apoptotic pathways, and suppresses cell growth of SK-N-AS neuroblastoma and SW620 metastatic colon cancer cells. Journal of ethnopharmacology. 2017; 207: 19-29.

132. Meng Z, Hou W, Zhou H, Zhou L, Chen H, Wu C. Therapeutic Considerations and Conjugated Polymer-Based Photosensitizers for Photodynamic Therapy. Macromolecular rapid communications. 2018; 39.

133. Zhang E, Xing R, Liu S, Qin Y, Li K, Li P. Advances in chitosan-based nanoparticles for oncotherapy. Carbohydrate polymers. 2019; 222: 115004

134. Baskaran R, Lee J, Yang SG. Clinical development of photodynamic agents and therapeutic applications. Biomaterials research. 2018; 22: 25. 